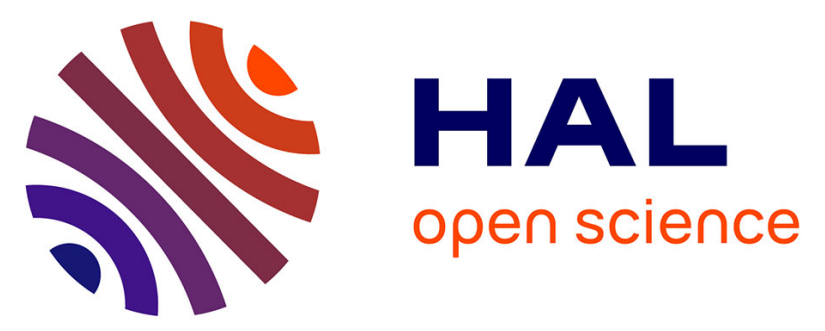

\title{
Modal parameter identification of stay cables from output-only measurements
}

\author{
Joseph Lardies, Minh-Ngi Ta
}

\section{To cite this version:}

Joseph Lardies, Minh-Ngi Ta. Modal parameter identification of stay cables from outputonly measurements. Mechanical Systems and Signal Processing, 2011, 25 (1), pp.133-150. 10.1016/j.ymssp.2010.05.020 . hal-02300174

\section{HAL Id: hal-02300174 \\ https://hal.science/hal-02300174}

Submitted on 26 Nov 2020

HAL is a multi-disciplinary open access archive for the deposit and dissemination of scientific research documents, whether they are published or not. The documents may come from teaching and research institutions in France or abroad, or from public or private research centers.
L'archive ouverte pluridisciplinaire HAL, est destinée au dépôt et à la diffusion de documents scientifiques de niveau recherche, publiés ou non, émanant des établissements d'enseignement et de recherche français ou étrangers, des laboratoires publics ou privés. 


\title{
Modal parameter identification of stay cables from output-only measurements
}

\author{
Joseph Lardies*, Minh-Ngi Ta \\ Institute FEMTO-ST; UMR 6174, Department of Applied Mechanics, L.M.A.R.C., University of Franche-Comté, 25 000, Besançon, France
}

Stay cables are one of the most critical structural components in modern cable-stayed bridges and the cable tension plays an important role in the construction, control and monitoring of cable-stayed bridges. We propose a time domain and a time-frequency domain approaches for modal parameter identification of stay cables using output-only measurements. The time domain approach uses the subspace algorithm which is improved with a new modal coherence indicator. The time-frequency approach uses the wavelet transform of signals which is improved with a new analyzing wavelet. The wavelet transform is applied to the free response of ambient vibration which is obtained using the random decrement technique. Two experiments of stay cables are presented. The first experiment concerns a stay cable in laboratory where the external load is applied through an impact hammer and the vibratory signals are acquired through four accelerometers. The second experiment concerns the Jinma cable-stayed bridge that connects Guangzhou and Zhaoqing in China. It is a single tower, double row cable-stayed bridge supported by 112 stay cables. Ambient vibration of each stay cable is carried out using accelerometers. From output-only measurements, the modal parameters of stay cables are extracted. Once the eigenfrequencies and the damping coefficients are obtained, the cable forces and the Scruton number are derived. In a continuous monitoring and modal analysis process, the tension forces and Scruton numbers could be used to assess the health of stay cables in cable-stayed bridges.

\section{Introduction}

Identification of modal parameters: eigenfrequencies, damping ratios and mode shapes from empirical data is of fundamental engineering importance in the dynamical analysis of stay cables, and more generally in the dynamical analysis of mechanical structures. These modal parameters will serve as reference to the finite element model updating, to perform structural health monitoring, damage detection and safety evaluation of structures. The extraction of modal parameters from frequency response function measurements requires knowledge of both: the input and the output from the system under test. However, in real operational conditions the input cannot be measured and an artificial excitation needs to be applied. This is very difficult to realize from an instrumental point of view and the resulting responses are often of poor quality, the useful signals being contaminated by the noise caused by the system's operation. Hence, in many cases, only operational response data are measurable and the system identification process will need to base itself on response only data. This identification based on the knowledge of output-only responses, without using excitation information, is

\footnotetext{
${ }^{*}$ Corresponding author

E-mail address: joseph.lardies@univ-fcomte.fr (J. Lardies).
} 
known as Operational Modal Analysis (OMA), also named as ambient or natural excitation or output-only modal analysis. OMA has drawn a great attention in mechanical and civil engineering community with applications for rotating machinery, wings or structures of aircraft during flight, wind tunnel testing, wind turbines, off-shore platforms, buildings, stadiums, towers, bridges, etc, which are under natural or environmental excitation. The principal advantages of OMA are the ease of use and the fast to conduct; since no artificial excitation equipment is required, the identification of modal properties of the whole system at representative working points, and finally, OMA can not only be utilized for structural analysis and design but also for vibration control and structural health monitoring. Note that in experimental tests in laboratory, where artificial excitations such as a swept sine, periodic chirp, impact or random forces are applied, OMA techniques can also be applied. Several OMA parameter identification algorithms are proposed in the literature [1-6] and these algorithms work in the frequency domain or in the time domain. In the frequency domain, the power spectral density of output responses is obtained using several signal processing techniques and natural frequencies are determined through a peak-picking procedure. However, there are limits with these methods in dealing with heavy damping and closeness of natural frequencies. The reason for the limitation is essentially modal interference and hence some individual modes and natural frequencies cannot be observed individually. In the time domain, correlation functions or covariance matrices between output responses are used to obtain state space matrices and extract modal parameters. We propose in this work a time domain and a time-frequency domain procedures to extract the modal parameters of vibrating systems from output-only measurements.

The first procedure is based on subspace algorithms, which use covariance matrices between output signals and are able to give estimates of high precision. They are constructed on the observability and controllability properties of linear time invarying systems and need linear algebra operations, such as the singular value decomposition (SVD) or QR factorization to filter noises, to estimate the state space matrices and the model order. However, all the time domain modal identification algorithms have a problem on model order estimation. When extracting physical modes the time domain algorithms generate spurious or computational modes to account for unwanted effects such as noise, leakage, residuals and nonlinearities. Therefore, it is necessary to completely identify and remove spurious modes. So far, there are many criteria such as MCF, MAmC and CEI that can be used to remove spurious modes and validate identified modal parameters [6-9]. Besides these criteria, the stabilization diagram which represents the variation of modal parameters with model order increments is also an effective tool to eliminate the non-structural modes. The original contribution of the paper is to propose a new modal indicator to distinguish structural and spurious modes. This modal indicator, named as Modal Coherence Indicator, is developed and implemented using the product of the modal observability coherence indicator and the modal controllability coherence indicator. In fact, this indicator describes the coherence between the modes of the identified state space model and the modes obtained from measured responses. This modal indicator is used to eliminate spurious modes. Two experimental examples show the performances of this indicator in modal parameter identification.

The second procedure used to identify modal parameters from output-only data is the wavelet transform (WT) [10-14]. The WT is a time-frequency representation and applied to the free response of a system, which allows the identification of eigenfrequencies, damping ratios and mode shapes. Indeed, the WT of a signal results in a complex valued form, whose modulus and phase can be related to the amplitude and phase modulation laws of the signal. For a linear system the modulus of the WT is related to the damping of the system and the phase of the WT to the natural frequency of the system. The WT representation in the time-frequency domain results is very useful when the analyzed signal is the free response of a multi-degree freedom system. In fact, the time-frequency maps allow the decay of each mode of the vibrating system to be followed separately from the others, by selecting the right frequency value corresponding to the mode of interest. Modes are then decoupled in the time-frequency domain and it is possible to follow the envelope decay and the phase variation in time of each mode and identify the modal parameters associated to the isolated mode. The estimation procedure using WT requires the free response of signals, however in OMA the measured signals are generally random. The ambient data are then transformed into free vibration data, before usage of the WT, by the application of the random decrement technique [15]. In this paper we use a new analyzing wavelet, named the modified Morlet wavelet. To obtain the optimal value of this analyzing wavelet we minimize the wavelet transform entropy and it is shown that this new analyzing wavelet improves the time-frequency resolution of the wavelet transform.

In this work the subspace method and the wavelet transform method are applied to study the dynamic behaviour of line cables. Two examples using real data are presented. The first experiment concerns a horizontal cable in laboratory where the external load is applied through an impact hammer and the vibratory signals are acquired through four accelerometers [16,17]. The second experiment concerns the Jinma cable-stayed bridge that connects Guangzhou and Zhaoqing in China [18]. It is a single tower, double row cable-stayed bridge supported by 112 stay cables. Ambient vibrations of each stay cable are carried out using accelerometers.

This paper is organised as follows. In Section 2 a mathematical model of the dynamic behaviour of transmission line cables and the subspace method are presented. The determination of the true structural modes is based upon a modal coherence indicator formed with identified and measured covariance matrices of signals. In Section 3 the wavelet transform is presented and to improve the results a modified Morlet wavelet function is introduced. In Section 4 experimental results in laboratory and in environmental conditions are presented. This paper is briefly concluded in Section 5. 


\section{A mathematical model and the subspace method}

\subsection{A mathematical model}

Study of vibrations of cables is similar to the analysis of a beam under the action of axial forces. This model is usually used to evaluate the behaviour of the cable submitted to the action of an external load such as the excitation due to the wind and to mechanical tension. The differential equation of cable motion is [19]

$$
E I \frac{\partial^{4} w(x, t)}{\partial x^{4}}+\rho S \frac{\partial^{2} w(x, t)}{\partial t^{2}}-P \frac{\partial^{2} w(x, t)}{\partial x^{2}}=f(x, t)
$$

where $E$ is the Young's modulus, $I$ the moment of inertia of the cable cross-section, $\rho$ the specific mass, $S$ the cross-sectional area, $P$ the axial load, $f(x, t)$ the external load, $w(x, t)$ the transversal displacement which is assumed to be small, $x$ the position along the cable and $t$ the time variable. For the free vibration the external load is zero and the solution of Eq. (1) is obtained by the method of separation of variables. The theoretical natural frequencies of vibration are then given by [19]

$$
f_{k}=\frac{\pi}{2 L^{2}}\left(\frac{E I}{\rho S}\right)^{1 / 2}\left(k^{4}+\frac{k^{2} P L^{2}}{\pi^{2} E I}\right)^{1 / 2}
$$

where $k$ is the free vibration mode number, and $L$ the length of the cable. We would like to approximate the desired partial differentiate equation solution $w(x, t)$ in a separable form as series expansion of time varying coefficients $q_{i}(t)$ and spatially varying basis functions $c_{i}(x)$

$$
\tilde{w}(x, t)=\sum_{i=1}^{n^{\prime}} q_{i}(t) c_{i}(x)
$$

where $\tilde{w}(x, t)$ is the approximation for $w(x, t)$. Assuming that the basis functions are known, the Galerkin procedure can then be used to specify the equations of motion for the coefficients $q_{i}(t)$. This method requires that the partial differentiate equation residual is orthogonal to each of the basis functions and Eq. (1) is then transformed in the following ordinary differential equation:

$$
\boldsymbol{M} \ddot{\boldsymbol{q}}+\boldsymbol{K q}=\mathbf{g}
$$

where

$$
\begin{aligned}
& M_{i j}=\int_{0}^{L} \rho S c_{i}(x) c_{j}(x) d x \\
& \left.K_{i j}=\int_{0}^{L} E I \frac{d^{2} c_{i}(x)}{d x^{2}} \frac{d^{2} c_{j}(x)}{d x^{2}}+P \frac{d c_{i}(x)}{d x} \frac{d c_{j}(x)}{d x}\right) d x \\
& g_{i}=\int_{0}^{L} c_{i}(x) f(x, t) d x
\end{aligned}
$$

In [16] Barbieri et al. used this procedure and a resolution of (4) by a FEM analysis is proposed. The basis functions $c_{i}(x)$ are computed with cubical Lagrangean finite elements and the natural frequencies of line cables are obtained. In this work, our purpose is to identify the modal parameters of a cable from response data only, using the signals of accelerometers placed in the cable, and to compare the theoretical results with experimental results.

For an $n^{\prime}$ degrees of freedom system with viscous damping, the motion equation is

$$
\boldsymbol{M} \ddot{\boldsymbol{q}}+\boldsymbol{C} \dot{\boldsymbol{q}}+\boldsymbol{K q}=\boldsymbol{f}
$$

where $\boldsymbol{M}$ is the mass matrix, and $\boldsymbol{C}, \boldsymbol{K}$ are the damping and stiffness matrices respectively. In [16] Barbieri et al. propose a procedure based on experimental and simulated data to identify eigenfrequencies of a transmission line cable. The experimental data are collected through accelerometers and the simulated data are obtained using the finite element method. In this work, we identify damping ratios of line cables from output-only measurements using the subspace method and the wavelet transform method.

\subsection{The subspace method}

The subspace identification method assumes that the dynamic behaviour of a structure excited by ambient forces can be described by a discrete time stochastic state space model $[1,3,6]$

$$
\begin{aligned}
& z_{\boldsymbol{k}+1}=\boldsymbol{A} \boldsymbol{z}_{\boldsymbol{k}}+\boldsymbol{w}_{\boldsymbol{k}} \text { state equation } \\
& \boldsymbol{y}_{\boldsymbol{k}}=\boldsymbol{C} \boldsymbol{z}_{\boldsymbol{k}}+\boldsymbol{v}_{\boldsymbol{k}} \text { observation equation }
\end{aligned}
$$


where $\boldsymbol{z}_{\boldsymbol{k}}$ is the unobserved state vector of dimension $n=2 n^{\prime}, \boldsymbol{y}_{\boldsymbol{k}}$ the $(m \times 1)$ vector of observations or measured output vector at discrete time instant $k, \boldsymbol{w}_{\boldsymbol{k}}, \boldsymbol{v}_{\boldsymbol{k}}$ are the white noise terms representing process noise and measurement noise together with the unknown inputs, it is assumed that the excitation effect appears in the disturbances $\boldsymbol{w}_{\boldsymbol{k}}$ and $\boldsymbol{v}_{\boldsymbol{k}}$, since the system input cannot be measured, $\boldsymbol{A}$ the $(n \times n)$ transition matrix describing the dynamics of the system and $\boldsymbol{C}$ the $(m \times n)$ output or observation matrix, translating the internal state of the system into observations. The stochastic identification problem deals with the determination of the two state space matrices $\boldsymbol{A}$ and $\boldsymbol{C}$ using output-only measurements.

The modal parameters of a vibrating system are obtained by applying the eigenvalue decomposition of the transition matrix $\boldsymbol{A}$

$$
\boldsymbol{A}=\Psi \Lambda \Psi^{-1}
$$

where $\boldsymbol{\Lambda}=\operatorname{diag}\left(\lambda_{i}\right), i=1,2, \ldots, n$, is the diagonal matrix containing the complex eigenvalues and $\boldsymbol{\Psi}$ contains the eigenvectors of $\boldsymbol{A}$ as columns. The eigenfrequencies $f_{i}$ and damping ratios $\zeta_{i}$ are obtained from the eigenvalues which are complex conjugate pair

$$
\begin{aligned}
f_{i} & =\frac{1}{4 \pi \Delta t} \sqrt{\left[\ln \left(\lambda_{i} \lambda_{i}^{*}\right)\right]^{2}+4\left[\arccos \left(\frac{\lambda_{i}+\lambda_{i}^{*}}{2 \sqrt{\lambda_{i} \lambda_{i}^{*}}}\right)\right]^{2}} \\
\zeta_{i} & =\sqrt{\frac{\left[\ln \left(\lambda_{i} \lambda_{i}^{*}\right)\right]^{2}}{\left[\ln \left(\lambda_{i} \lambda_{i}{ }^{*}\right)\right]^{2}+4\left[\arccos \left(\frac{\lambda_{i}+\lambda_{i}^{*}}{2 \sqrt{\lambda_{i} \lambda_{i}^{*}}}\right)\right]^{2}}}
\end{aligned}
$$

with $\Delta t$ the sampling period of analyzed signals.

The mode shapes evaluated at the sensor locations are the columns of the matrix $\tilde{\boldsymbol{C}}$ obtained by multiplying the output matrix $\boldsymbol{C}$ with the matrix of eigenvectors $\boldsymbol{\Psi}$ :

$$
\tilde{\boldsymbol{C}}=C \boldsymbol{\Psi}
$$

Our purpose is to determine the transition matrix $\boldsymbol{A}$ and the output matrix $\boldsymbol{C}$ in order to obtain the modal parameters of the vibrating system. Define the $(m f x 1)$ and $(m p x 1)$ future and past data vectors as $\boldsymbol{y}_{k}^{+}=\left[\boldsymbol{y}_{K}^{T}, \boldsymbol{y}_{k+1}^{T}, \ldots, \boldsymbol{y}_{k+f-1}^{T}\right]^{T}$ and $\boldsymbol{y}_{k}^{-}=\left[\boldsymbol{y}_{k}^{T}, \boldsymbol{y}_{k+1}^{T}, \ldots, \boldsymbol{y}_{k-p+1}^{T}\right]^{\mathrm{T}}$. The (mfxmp) covariance matrix between the future and the past is given by

$$
\boldsymbol{H}=E\left[\boldsymbol{y}_{k}^{+} \boldsymbol{y}_{k}^{-T}\right]=\left[\begin{array}{cccc}
\boldsymbol{R}_{\mathbf{1}} & \boldsymbol{R}_{\mathbf{2}} & \cdot & \boldsymbol{R}_{\boldsymbol{p}} \\
\boldsymbol{R}_{\mathbf{2}} & \boldsymbol{R}_{\mathbf{3}} & \cdot & \boldsymbol{R}_{\boldsymbol{p}+\mathbf{1}} \\
\cdot & \cdot & \cdot & \cdot \\
\boldsymbol{R}_{\boldsymbol{f}} & \boldsymbol{R}_{\boldsymbol{f}+\mathbf{1}} & \cdot & \boldsymbol{R}_{\boldsymbol{f}+\boldsymbol{p}-\mathbf{1}}
\end{array}\right]
$$

where $E$ denotes the expectation operator and the superscript $T$ the transpose operation. $\boldsymbol{H}$ is the block Hankel matrix formed with the $(m x m)$ individual theoretical auto-covariance matrices $\boldsymbol{R}_{\boldsymbol{i}}=E\left[\boldsymbol{y}_{k+i} \boldsymbol{y}_{k}^{T}\right]=\boldsymbol{C} \boldsymbol{A}^{\mathrm{i}-1} \boldsymbol{G}$ with $\boldsymbol{G}=E\left[\boldsymbol{x}_{k+1} \boldsymbol{y}_{k}^{T}\right]$.

In practice, the auto-covariance matrices are estimated from $N$ data points and are computed by $\boldsymbol{R}_{\boldsymbol{i}}=N^{-1} \sum_{k=1}^{N-1} \boldsymbol{y}_{k+i} \boldsymbol{y}_{k}^{T}$; $i=0,1, \ldots, p+f$ and with these estimated auto-covariance matrices we form the block Hankel matrix $\boldsymbol{H}$. In order to identify the transition matrix $\boldsymbol{A}$ and the output influence matrix $\boldsymbol{C}$ two matrix factorizations of $\boldsymbol{H}$ are employed. The first factorization uses the singular value decomposition (SVD) of $\boldsymbol{H}$

$$
\boldsymbol{H}=U \boldsymbol{\Sigma} \boldsymbol{V}^{T}=\boldsymbol{U} \Sigma^{1 / 2} \Sigma^{1 / 2} \boldsymbol{V}^{T}
$$

with $\boldsymbol{U}^{T} \boldsymbol{U}$ and $\boldsymbol{V}^{T} \boldsymbol{V}$ identity matrices and $\Sigma$ a diagonal matrix of singular values. The second factorization of the block Hankel matrix $\boldsymbol{H}$ considers its ( $m f x n$ ) observability and ( $n x m p)$ controllability matrices, $\boldsymbol{O}$ and $\boldsymbol{K}$, as

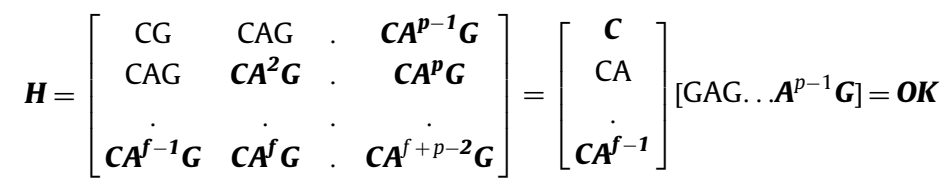

By identification, the observability matrix $\boldsymbol{O}$ is expressed in terms of $\boldsymbol{C}$ and $\boldsymbol{A}$ and the controllability matrix $\boldsymbol{K}$ is expressed in terms of $\boldsymbol{G}$ and $\boldsymbol{A}$.

The two factorizations of the block Hankel matrix are equated to give

$$
\boldsymbol{H}=\boldsymbol{U} \Sigma^{1 / 2} \Sigma^{1 / 2} \boldsymbol{V}^{T}=\mathbf{O K}
$$

implying $\boldsymbol{O}=\boldsymbol{U} \Sigma^{\mathbf{1} / \mathbf{2}}$ and $\boldsymbol{K}=\Sigma^{\mathbf{1 / 2}} \boldsymbol{V}^{\boldsymbol{T}}$. Properties of the observability and controllability matrices are used to determine the transition matrix $\boldsymbol{A}$. Let $\boldsymbol{O}^{\downarrow}(m(f-1) \times n)$ be the shifted matrix obtained by deleting the last block row of $\boldsymbol{O}$ and let $\boldsymbol{O}^{\uparrow}$ $(m(f-1) \times n)$ be the shifted matrix obtained by deleting the first block row of $\boldsymbol{O}$. It is easy to show that

$$
\boldsymbol{O}^{\uparrow}=\boldsymbol{O}^{\downarrow} \boldsymbol{A} \text { or }\left(\boldsymbol{U} \Sigma^{1 / 2}\right)^{\uparrow}=\left(\boldsymbol{U} \Sigma^{1 / 2}\right)^{\downarrow} \boldsymbol{A}
$$


Properties of shifting operators “ $\uparrow$ ” and “ $\downarrow$ ” are exploited: let $\boldsymbol{\Delta}$ and $\boldsymbol{\Gamma}$ be two (axb) and (bxc) matrices, we have: $(\Delta \boldsymbol{\Gamma})^{\uparrow}=\Delta^{\uparrow} \boldsymbol{\Gamma}$ and $(\Delta \Gamma)^{\downarrow}=\Delta^{\downarrow} \boldsymbol{\Gamma}$, consequently

$$
\left(\boldsymbol{U}^{\uparrow} \Sigma^{1 / 2}\right)=\left(\boldsymbol{U}^{\downarrow} \Sigma^{1 / 2}\right) \boldsymbol{A}
$$

and the transition matrix is

$$
\boldsymbol{A}=\left(\boldsymbol{U}^{\downarrow} \Sigma^{1 / 2}\right)+\left(\boldsymbol{U}^{\uparrow} \Sigma^{1 / 2}\right)=\Sigma^{-/ 2}\left(\boldsymbol{U}^{\downarrow}\right)^{+} \boldsymbol{U}^{\uparrow} \Sigma^{1 / 2}
$$

where ()$^{+}$represents the pseudo inverse of a matrix, and the eigenvalues of the transition matrix are given by

$$
\lambda(\boldsymbol{A})=\left[\left(\boldsymbol{U}^{\downarrow}\right)^{+} \boldsymbol{U}^{\uparrow}\right]
$$

Two methods can be used to determine the output matrix $\boldsymbol{C}$. In the first method, let $\boldsymbol{H}_{\mathbf{1} \boldsymbol{R}}$ be the first block row of the block Hankel matrix $\boldsymbol{H}$. It is easy to show that

$$
\boldsymbol{H}_{1 \boldsymbol{R}}=\left[\boldsymbol{R}_{1} \boldsymbol{R}_{2} \ldots \boldsymbol{R}_{p}\right]=\mathrm{CK}
$$

and the estimate of $\boldsymbol{C}$ is obtained using the pseudo-inverse of $\boldsymbol{K}$

$$
\boldsymbol{C}=\boldsymbol{H}_{1 \boldsymbol{R}} \boldsymbol{K}^{+}=\boldsymbol{H}_{1 \boldsymbol{R}} \boldsymbol{V} \Sigma^{-1 / 2}
$$

In the second method we observe that $\boldsymbol{C}$ is only the first block row of the observability matrix $\boldsymbol{O}$

$$
\boldsymbol{C}=\boldsymbol{O}_{1 \boldsymbol{R}}=\left(\boldsymbol{U} \Sigma^{1 / 2}\right)_{1 \boldsymbol{R}}
$$

With estimates of $\boldsymbol{A}$ and $\boldsymbol{C}$ in hand we compute the eigenvalues and eigenvectors of $\boldsymbol{A}$, and from (12-14) we identify eigenfrequencies, damping ratios and mode shapes of the vibrating system.

However, all the subspace modal identification algorithms have a serious problem of model order determination. When extracting physical or structural modes, subspace algorithms always generate spurious or computational modes to account for unwanted effects such as noise, leakage, residuals, nonlinearity's ... Furthermore, weakly excited modes often require relatively high numbers of assumed modes to be properly identified. For these reasons, the assumed number of modes, or model order, is incremented over a wide range of values and we plot the stability diagram. The stability diagram involves tracking the estimates of eigenfrequencies and damping ratios as a function of model order. As the model order is increased, more and more modal frequencies and damping ratios are estimated, hopefully, the estimates of the physical modal parameters stabilize as the correct model order is reached. For modes which are very active in the measured data, the modal parameters stabilize at a very low model order. For modes that are poorly excited, the modal parameters may not stabilize until a very high model order is chosen. Nevertheless, the non-physical modes, called spurious modes, do not stabilize at all during this process and can be sorted out of the modal parameters. A criterion based on the modal coherence of measured and identified modes is used to detect these spurious modes and remove them from the model.

\subsection{The modal coherence indicator}

In first the modal observability coherence indicator is derived. In modal coordinates the identified covariance matrices are

$$
\boldsymbol{R}_{i}=\tilde{\boldsymbol{C}} \Lambda^{i-} 1 \tilde{\boldsymbol{G}}
$$

where $\tilde{\boldsymbol{C}}=\boldsymbol{C} \boldsymbol{\Psi}$ is the identified matrix of mode shapes or output matrix in modal coordinates and $\tilde{\boldsymbol{G}}=\boldsymbol{\Psi}^{-1} \boldsymbol{G}$ the identified cross-covariance matrix between the state and the output. In modal coordinates the block Hankel matrix is

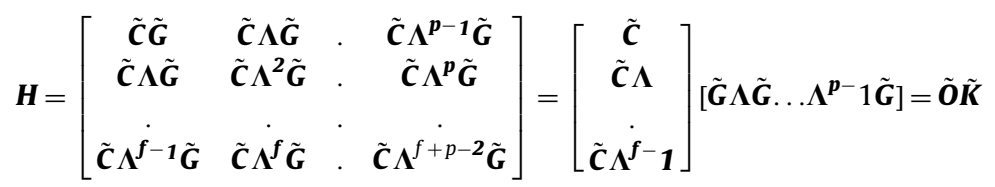

By identification, the observability matrix $\tilde{\boldsymbol{O}}$ is expressed in terms of $\tilde{\boldsymbol{C}}$ and $\boldsymbol{\Lambda}$ and the controllability matrix $\tilde{\boldsymbol{K}}$ is expressed in terms of $\tilde{\boldsymbol{G}}$ and $\boldsymbol{\Lambda}$.

We note $\tilde{\mathbf{q}}_{\mathbf{1}}^{\boldsymbol{o}}, \tilde{\mathbf{q}}_{2}^{\boldsymbol{o}}, \ldots, \tilde{\boldsymbol{q}}_{\boldsymbol{n}}^{\boldsymbol{o}}$ the columns of the identified observability matrix $\tilde{\boldsymbol{O}}$. It is easy to show that the $k$ th column of $\tilde{\boldsymbol{O}}$ is the $(m f \times 1)$ vector obtained as

$$
\tilde{\mathbf{q}}_{\boldsymbol{k}}^{\boldsymbol{o}}=\left[\begin{array}{c}
\tilde{\boldsymbol{c}}_{\boldsymbol{k}} \\
\lambda_{k} \tilde{\boldsymbol{c}}_{\boldsymbol{k}} \\
\cdot \\
\lambda_{k}^{f-1} \tilde{\boldsymbol{c}}_{\boldsymbol{k}}
\end{array}\right]
$$

with $\tilde{\boldsymbol{c}}_{\boldsymbol{k}}(m \times 1)$ being the $k$ th column vector of the matrix $\tilde{\boldsymbol{C}}$. The sequence $\left\{\tilde{\boldsymbol{q}}_{\boldsymbol{k}}^{\boldsymbol{o}}\right\}$ is called the identified modal time story for the $k$ th mode obtained from the identified observability matrix. It represents the temporal contribution of the $k$ th mode associated with the output vector $\tilde{\boldsymbol{c}}_{\boldsymbol{k}}$ to the identified observability matrix. The Hankel matrix can be decomposed by using 
the singular value decomposition to become

$$
\boldsymbol{H}=\left(\boldsymbol{U} \Sigma^{1 / 2} \boldsymbol{\Psi}\right)\left(\boldsymbol{\Psi}^{-1} \Sigma^{1 / 2} \boldsymbol{V}^{T}\right)=\overline{\boldsymbol{O}} \overline{\boldsymbol{K}}
$$

where the matrices $\overline{\boldsymbol{O}}(m f x n)$ and $\overline{\boldsymbol{K}}(n x m p)$ calculated from measured covariance matrices are called the measured observability and controllability matrices. The measured observability matrix is obtained from Eq. (29)

$$
\overline{\boldsymbol{O}}=\boldsymbol{U} \Sigma^{1 / 2} \boldsymbol{\Psi}=\left[\overline{\boldsymbol{q}}_{\mathbf{1}}^{\mathbf{o}} \overline{\boldsymbol{q}}_{\mathbf{2}}^{\boldsymbol{o}} \ldots \overline{\boldsymbol{q}}_{\boldsymbol{n}}^{\boldsymbol{o}}\right]
$$

where $\overline{\boldsymbol{q}}_{\boldsymbol{k}}^{\boldsymbol{o}}$ is the $k$ th $(m f x 1)$ column vector of the measured observability matrix $\overline{\boldsymbol{0}}$. The modal observability coherence indicator is defined as the magnitude of the normalized dot product between the vectors $\tilde{\mathbf{q}}_{\boldsymbol{k}}^{\boldsymbol{o}}$ and $\overline{\boldsymbol{q}}_{\boldsymbol{k}}^{\boldsymbol{o}}$

$$
\gamma_{k}^{o}=\frac{\left|\tilde{\boldsymbol{q}}_{k}^{o^{*}} \overline{\mathbf{q}}_{k}^{o}\right|}{\left(\left|\tilde{\boldsymbol{q}}_{k}^{o^{*}} \tilde{\mathbf{q}}_{k}^{o}\right|\left|\overline{\boldsymbol{q}}_{k}^{o^{*}} \overline{\boldsymbol{q}}_{k}^{o}\right|\right)^{1 / 2}}
$$

with * indicating complex conjugate transposition.

In a similar way we define the modal controllability coherence indicator. We note $\tilde{\mathbf{q}}_{1}^{c}, \tilde{\mathbf{q}}_{2}^{c}, \ldots, \tilde{\boldsymbol{q}}_{n}^{c}$ the rows of the identified controllability matrix $\tilde{\boldsymbol{K}}$. It is easy to show that the $k$ th row of $\tilde{\boldsymbol{K}}$ is the $(1 \mathrm{xmp})$ row vector obtained as

$$
\tilde{\boldsymbol{q}}_{k}^{c}=\left[\tilde{\boldsymbol{g}}_{k} \lambda_{k} \tilde{\boldsymbol{g}}_{k} \lambda_{k}^{2} \tilde{\boldsymbol{g}}_{k} \ldots \lambda_{k}^{p-1} \tilde{\boldsymbol{g}}_{k}\right]
$$

with $\tilde{\boldsymbol{g}}_{k}(1 \mathrm{xm})$ being the $k$ th row vector of the matrix $\tilde{\boldsymbol{G}}$. The sequence $\left\{\tilde{\boldsymbol{q}}_{k}^{c}\right\}$ is called the identified modal time story for the $k$ th mode obtained from the identified controllability matrix. It represents the temporal contribution of the $k$ th mode associated with the vector $\tilde{\boldsymbol{g}}_{k}$ to the identified controllability matrix. The measured controllability matrix is obtained from Eq. (29)

$$
\overline{\mathbf{K}}=\boldsymbol{\Psi}^{-1} \Sigma^{1 / 2} \boldsymbol{V}^{T}=\left[\begin{array}{c}
\overline{\boldsymbol{q}}_{1}^{c} \\
\overline{\mathbf{q}}_{2}^{c} \\
\cdot \\
\overline{\mathbf{q}}_{n}^{c}
\end{array}\right]
$$

where $\overline{\boldsymbol{q}}_{k}^{c}$ is the $k$ th $(1 \mathrm{xmp})$ row vector of the measured controllability matrix $\overline{\mathbf{K}}$. The modal controllability coherence indicator is defined as the magnitude of the normalized dot product between the vectors $\tilde{\boldsymbol{q}}_{k}^{c}$ and $\overline{\boldsymbol{q}}_{k}^{c}$

$$
\gamma_{k}^{c}=\frac{\left|\tilde{\boldsymbol{q}}_{k}^{c} \overline{\boldsymbol{q}}_{k}^{c^{*}}\right|}{\left(\left|\tilde{\boldsymbol{q}}_{k}^{c} \tilde{\boldsymbol{q}}_{k}^{c^{*}}\right|\left|\overline{\boldsymbol{q}}_{k}^{c} \overline{\boldsymbol{q}}_{k}^{c^{*}}\right|\right)^{1 / 2}}
$$

Finally, the modal coherence indicator is defined for $k=1,2 \ldots, n$ as $\gamma_{k}=\gamma_{k}^{o} \gamma_{k}^{c}$. Given a state space model and a set of measured covariance matrices, the modal coherence indicator describes the correlation between each mode of the identified state space model and the modes directly inferred from the measured signal and thus serve as a distributed model quality measure. This indicator generalizes the modal amplitude coherence (MAC) indicator introduced by Juang [7]. The MAC uses only the controllability matrix of the estimated state-space model, and is only defined for state space models derived from the eigensystem realization algorithm (ERA), where multi-input and multi-output impulse response functions are used to form a Markov parameter sequence. Here we generalize the indicator to operational modal analysis where only multi-output data are used to form covariance matrices.

\section{The wavelet transform}

\subsection{Background and definitions}

There are several time-frequency representations such as the Short Time Fourier Transform (STFT), the Wigner-Vile Distribution (WVD) and the Wavelet Transform (WT). In general, the STFT has some limitations in analyzing a signal that varies with an instantaneous frequency because its time-frequency resolution is fixed. The WVD has a better timefrequency resolution than the STFT but, despite of its remarkable properties, the WVD has limited applications because it contains the cross-talk effects. The WT can overcome the limitations of the STFT and the WVD because the time-frequency resolution of the WT can be controlled adaptively. Recently, several studies have been carried out using the WT to identify the modal parameters of vibrating systems [11-14] and the aim of the paper is to propose a new analyzing wavelet to identify the modal characteristics of stay cables.

The wavelet transform (WT) gives time and frequency information about the analyzed data. For all functions $x(t)$ satisfying the condition $\int_{-\infty}^{+\infty}|x(t)|^{2} d t<\infty$, which implies that $x(t)$ decays to zero at $\pm \infty$, the wavelet transform of $x(t)$ is defined as $[10-14]$

$$
\left(W_{\psi} x\right)(a, b)=\frac{1}{\sqrt{a}} \int_{-\infty}^{+\infty} x(t) \psi^{*}\left(\frac{t-b}{a}\right) d t
$$


where $\psi(t)$ is an analyzing function called mother wavelet, $a$ the dilatation or scale parameter defining the analyzing window stretching and $b$ the translation parameter localising the wavelet function in the time domain. The WT represents the correlation between the signal $x(t)$ and a scaled version of the function $\psi(t)$ and the idea of the WT is to decompose a signal $x(t)$ into wavelet coefficients using the basis of wavelet functions. The decomposition is obtained locally at different time windows and frequency bands. The size of the time window is controlled by the translation parameter $b$ while the length of the frequency band is controlled by the dilatation parameter $a$. Hence, one can examine the signal at different time windows and frequency bands by controlling translation and dilatation.

Since the wavelet transform is a linear representation of a signal, it follows that the WT of $P$ signals is

$$
\left.W_{\psi} \sum_{i=1}^{P} x_{i}\right)(a, b)=\sum_{i=1}^{P}\left(W_{\psi} x_{i}\right)(a, b)
$$

this property is convenient for the analysis of multi-component signals.

A number of different analyzing functions have been used in the wavelet analysis. One of the most known and widely used is the Morlet wavelet defined in the time domain as [10-12]

$$
\psi(t)=e^{j \omega_{0} t} e^{-t^{2} / 2}
$$

where $\omega_{0}$ is the wavelet frequency. The dilated version of the Fourier transform is

$$
\Psi(a \omega)=\sqrt{2 \pi} e^{-\frac{1}{2}\left(a \omega-\omega_{0}\right)^{2}}
$$

In practice the value of $\omega_{0}$ is chosen $\omega_{0}=2 \pi / \sqrt{2 \log (2)}$ which meets approximately the requirements of admissibility condition [10]. Note that $\Psi(a \omega)$ is maximum at the central frequency $\omega_{c}=\omega_{0} / a$ and the Morlet wavelet can be viewed as a linear bandpass filter whose bandwidth is proportional to $1 / a$ or to the central frequency. Thus, the value of the dilatation parameter $a$ at which the wavelet filter is focused on the wavelet frequency can be determined from $a=\omega_{0} / \omega_{\mathrm{c}}$.

For a given value of the dilatation parameter $a$ the spectrum of the Morlet wavelet has a fixed bandwidth. If the analyzed frequency is important the dilatation parameter becomes small and the spectrum of the Morlet wavelet function is wide. There is then a bad spectral resolution. An alternative is to modify the Morlet wavelet function by introduction of a parameter $N$ which controls the shape of the basic wavelet: this parameter balances the time resolution and frequency resolution of the Morlet wavelet. The modified Morlet wavelet function used in this work is

$$
\psi(t)=e^{j \omega_{0} t} e^{-t^{2} / N}
$$

with $N>0$ and whose dilated version of its Fourier transform is

$$
\Psi(a \omega)=\sqrt{N \pi} e^{-\frac{N}{4}\left(a \omega-\omega_{0}\right)^{2}}
$$

The wavelet filter central frequency is $\omega_{c}=\omega_{0} / a$ and gives then a relation between the scale parameter $a$ and the central frequency of the modified Morlet wavelet.

When $N$ tends to infinity the modified Morlet wavelet tends to $e^{j \omega_{0} t}$, which has the finest frequency resolution allowing a better resolution of closely spaced modes, but at the expense of time resolution. Indeed, increasing $N$ will increase the frequency resolution but it decreases the time resolution. In other words, an important value of $N$ gives a narrower spectrum allowing a better resolution of closely spaced modes, but at the expense of time resolution, so, there always exists an optimal $N$ that has the best time-frequency resolution for a certain signal localized in the time-frequency plane. This modified Morlet wavelet function offers a better compromise in terms of localization, in both time and frequency for a signal, than the traditionally Morlet wavelet function. The optimal value of $N$ is obtained by minimizing the entropy of the wavelet transform.

\subsection{The wavelet transform entropy}

We assume that the signal $x(t)$ is given by sampled values $\{x(q)\}, q=1,2 \ldots, Q$. The total energy of this sequence is $\sum_{q}|x(q)|^{2}$ and the values

$$
p(q)=\frac{|x(q)|^{2}}{\sum_{q}|x(q)|^{2}}
$$

give the probability distribution of the signal's energy in the time domain. Furthermore, in the wavelet multi-resolution analysis of the time series $\{x(q)\}$ the energy for each scale $a_{i}$ is

$$
E_{a i}=\sum_{j}\left|W\left(a_{i}, b_{j}\right)\right|^{2}
$$

$E_{a i}$ is then obtained with a set of wavelet coefficients over a number of translations $b_{j}$, given a particular scale $a_{i}$. As a consequence, the total energy can be obtained by $E_{\text {total }}=\sum_{i} E_{\mathrm{ai}}$. Then the normalized values, $p_{a i}=E_{a i} / E_{\text {total }}$, which represent the relative wavelet energy for $i=1,2, \ldots, M$ define by scale the probability distribution of the energy. Clearly, $\sum_{i} p_{\mathrm{ai}}=1$ and the distribution $\left\{p_{a i}\right\}$ can be considered as a time-scale density. The Shannon entropy [20] gives a useful criteria for 
analyzing and comparing theses distributions, since it provides a measure of the information of any distribution. Following the definition of entropy given by Shannon, we define the time varying wavelet entropy as

$$
W E=-\sum_{i} p_{\mathrm{ai}} \log p_{\mathrm{ai}}
$$

which measures the degree of disorder or unpredictability of energy in each wavelet transform. Using the modified Morlet wavelet there exists an optimal value of the parameter $N$ obtained by the minimisation of the wavelet entropy.

\subsection{Application of the wavelet transform to modulated signals}

Consider the case of a signal $x(t)$ modulated in amplitude

$$
x(t)=A(t) \cos (\varphi(t))
$$

if $x(t)$ is assumed to be asymptotic, the WT of $x(t)$ can be obtained by means of asymptotic techniques and can be expressed as $[10,11]$

$$
\left(W_{\psi} x\right)(a, b)=\frac{\sqrt{a}}{2} A(b) \Psi^{*}(a \dot{\varphi}(b)) e^{j \varphi(b)}
$$

the point indicating a derivative. The dilatation parameter can be calculated in order to maximize $\Psi^{*}(a \dot{\varphi}(b))$, that is using the Morlet wavelet (or modified Molet wavelet) for the dilatation $a(b)=\omega_{0} / \dot{\varphi}(b)$. The wavelet transform is essentially concentrated in a neighbourhood of a curve given by $a(b)$ called the ridge of the WT.

Consider now the free response of a viscously damped single degree of freedom system

$$
x(t)=B e^{-\zeta \omega_{n} t} \cos \left(\omega_{d} t+\chi_{0}\right)
$$

with $B$ the residue magnitude, $\omega_{n}$ the undamped natural frequency, $\omega_{d}=\omega_{n} \sqrt{1-\zeta^{2}}$ the damped natural frequency, $\zeta$ the viscous damping ratio. If the system is underdamped, that is if the damping ratio is smaller than 1 , (in general $0<\zeta<<<1$, so $\left.\omega_{d} \approx \omega_{n}\right)$ the signal $x(t)$ can be considered asymptotic, and therefore the results obtained previously can be used considering

$$
\begin{aligned}
& A(t)=B e^{-\zeta \omega_{n} t} \\
& \varphi(t)=\omega_{d} t+\chi_{0} \Rightarrow \dot{\varphi}(t)=\omega_{d}
\end{aligned}
$$

The WT of the damped sinusoid is

$$
\left(W_{\psi} x\right)(a, b)=\frac{\sqrt{a}}{2} B e^{-\zeta \omega_{n} b} \Psi\left(a \omega_{d}\right) e^{j\left(\omega_{d} b+\chi_{0}\right)}
$$

For a fixed value $a_{0}$ of the dilatation parameter the logarithm of the WT amplitude is

$$
\ln \left|\left(W_{\psi} x\right)\left(a_{0}, b\right)\right|=-\zeta \omega_{n} b+\ln \left(\frac{\sqrt{a_{0}}}{2} B\left|\Psi^{*}\left(a_{0} \omega_{d}\right)\right|\right)
$$

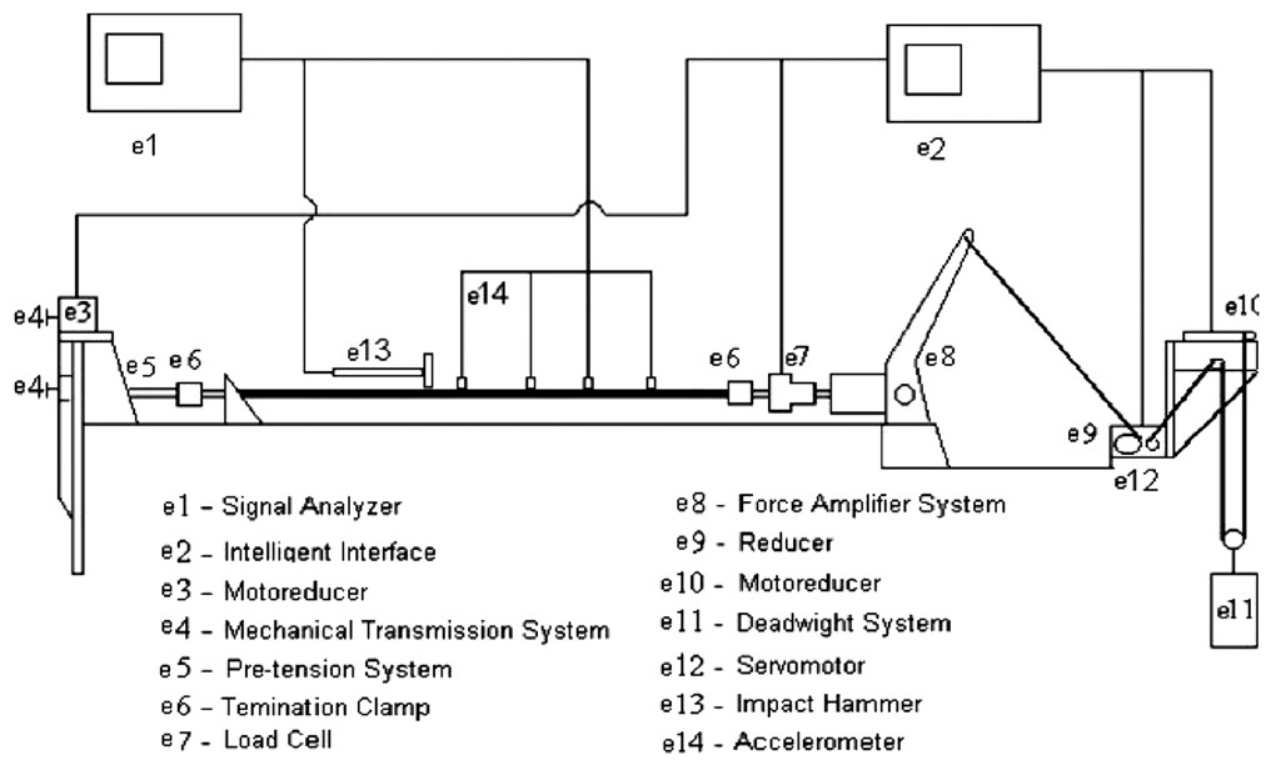

Fig. 1. Schematic view of the testing line cable in laboratory. 
Thus the decay rate $\sigma=\omega_{n} \zeta$ of the signal can be estimated from the slope of the straight line of the logarithm of the wavelet transform modulus. The WT phase is given by

$$
\operatorname{Arg}\left(W_{\psi}\left(a_{0}, b\right)\right)=\omega b+\chi_{0} \Rightarrow \frac{d}{d b} \operatorname{Arg}\left(W_{\psi}\left(a_{0}, b\right)\right)=\omega_{d}
$$

and the plot of $\frac{d}{d b} \operatorname{Arg}\left(W_{\psi}\left(a_{0}, b\right)\right)$ should be constant in time and equal to the damped natural frequency $\omega_{d}$.

The damping ratio and eigenfrequency estimation procedures, based on the wavelet transform presented above, can be extended to multi-degrees of freedom systems by selecting the right value of the dilatation parameter $a_{i}$ corresponding to the mode of interest. Consider now the free response of a $P$ degrees of freedom system

$$
x(t)=\sum_{i=1}^{P} B_{i} e^{-\zeta_{i} \omega_{n i} t} \cos \left(\omega_{d i} t+\chi_{0 i}\right)
$$

where $B_{i}$ is the residue magnitude, $\zeta_{i}$ the damping ratio, $\omega_{n i}$ the undamped natural frequency and $\omega_{d i}$ the damped natural frequency associated to the $i$ th mode. From Eq. (49), the wavelet transform of the multi-degrees of freedom system is

$$
\left.W_{\psi} \sum_{i=1}^{P} x_{i}\right)(a, b)=\frac{\sqrt{a}}{2} \sum_{i=1}^{P} B_{i} e^{-\zeta_{i} \omega_{n i} b} \Psi^{*}\left(a \omega_{d i}\right) e^{j\left(\omega_{d i} b+\chi_{0 i}\right)}
$$
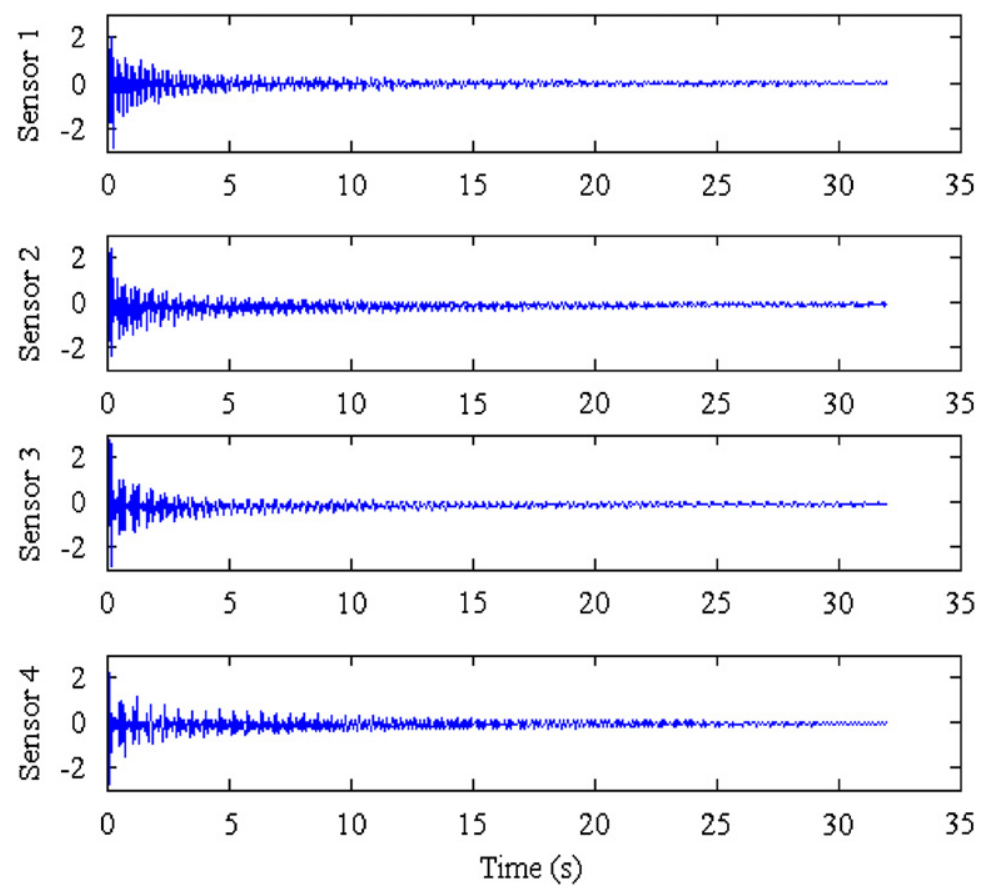

Fig. 2. Time response of accelerometers (amplitude $\left(\mathrm{mm} \mathrm{s}^{-2}\right)$ versus time (s)).

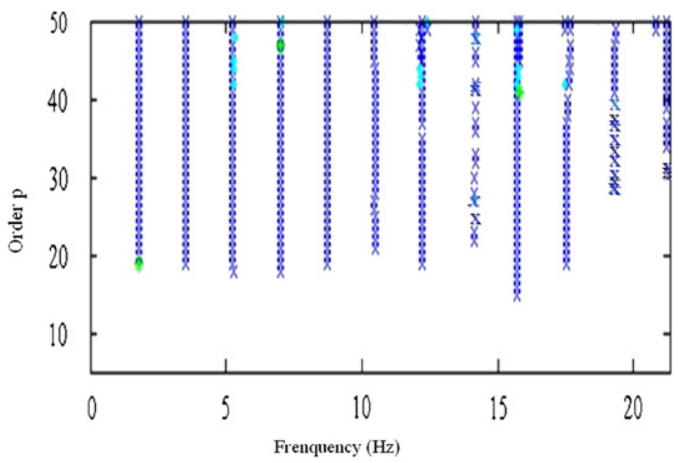

Fig. 3. Stabilization diagram on eigenfrequencies for the line cable in laboratory. 
The wavelet transform is a signal decomposition procedure working as a filter in the time-frequency domain: it analyzes a signal only locally at windows defined by the wavelet. Thus, multi-degrees of freedom system can be decoupled into single degrees of freedom. For a fixed value of the dilatation parameter $\left(a=a_{i}\right)$, which maximizes $\psi^{*}\left(a \omega_{d}\right)$, only the mode associated with $a_{i}$ gives a relevant contribution in the wavelet transform, while all the other terms are negligible. Thus the wavelet transform of each separated mode $i=1,2, \ldots, P$ becomes

$$
\left(W_{\psi} x\right)\left(a_{i}, b\right)=\frac{\sqrt{a_{i}}}{2} B_{i} e^{-\zeta_{i} \omega_{n i} b} \Psi^{*}\left(a_{i} \omega_{d i}\right) e^{j\left(\omega_{d i} b+\chi_{0 i}\right)}
$$

Clearly, the wavelet transform offers a decoupling of multi-degrees of freedom systems into single modes. However, Eq. (54) is true under the assumption of vanishing $\Psi^{*}\left(a_{i} \omega_{d i}\right)$ outside the interval $\left[f_{i}-\Delta f_{\psi} / a_{i}, f_{i}+\Delta f_{\psi} / a_{i}\right]$, that is, if none of the other frequencies of the system except $f_{i}$ and more likely if neither $f_{i-1}$ or $f_{i+1}$ belongs to the interval $\left[f_{i}-\Delta f_{\psi} / a_{i}, f_{i}+\Delta f_{\psi} / a_{i}\right]$. The resolution of the wavelet transform is good enough to separate the $i$ th mode from the neighbouring modes. Using (54) associated with (50) and (51), it is possible to follow the amplitude and phase variations in the time domain of each modal component and to estimate the corresponding damping ratio and eigenfrequency associated to the isolated mode.

When time responses recorded from several points of the system are available, amplitude and phase relationships between the different degrees of freedom can be obtained through the WT analysis and the $i$ th mode shape of the system can be evaluated by determining the WT of all sensors at the corresponding ith frequency. Let $\left(W_{\psi} x_{k}\right)\left(a_{i}, b\right)$ and $\left(W_{\psi} x_{r}\right)\left(a_{i}, b\right)$ be the WT of signals at point $k$ and at a reference point $r$ evaluated for the dilatation parameter $a_{i}$ corresponding to the $i$ th

Table 1

Natural frequencies and damping ratios of the experimental cable using the subspace method.

\begin{tabular}{|c|c|c|c|c|c|c|c|c|c|}
\hline \multirow[t]{2}{*}{ Modes } & \multirow[t]{2}{*}{ Theoretical frequency $(\mathrm{Hz})$} & \multicolumn{2}{|c|}{ Sensor 1,2} & \multicolumn{2}{|c|}{ Sensor 2} & \multicolumn{2}{|c|}{ Sensor 4} & \multicolumn{2}{|c|}{ Sensor $1-4$} \\
\hline & & $f(\mathrm{~Hz})$ & $\zeta(\%)$ & $f(\mathrm{~Hz})$ & $\zeta(\%)$ & $f(\mathrm{~Hz})$ & $\zeta(\%)$ & $f(\mathrm{~Hz})$ & $\zeta(\%)$ \\
\hline 1 & 1.765 & 1.776 & 0.509 & 1.774 & 0.518 & 1.773 & 0.507 & 1.775 & 0.520 \\
\hline 2 & 3.530 & 3.517 & 0.275 & 3.518 & 0.262 & 3.517 & 0.251 & 3.518 & 0.265 \\
\hline 3 & 5.295 & 5.255 & 0.282 & 5.256 & 0.293 & 5.256 & 0.261 & 5.255 & 0.265 \\
\hline 4 & 7.060 & 7.016 & 0.170 & 7.016 & 0.161 & 7.016 & 0.163 & 7.016 & 0.168 \\
\hline 5 & 8.825 & 8.729 & 0.174 & 8.729 & 0.181 & 8.732 & 0.174 & 8.730 & 0.175 \\
\hline
\end{tabular}
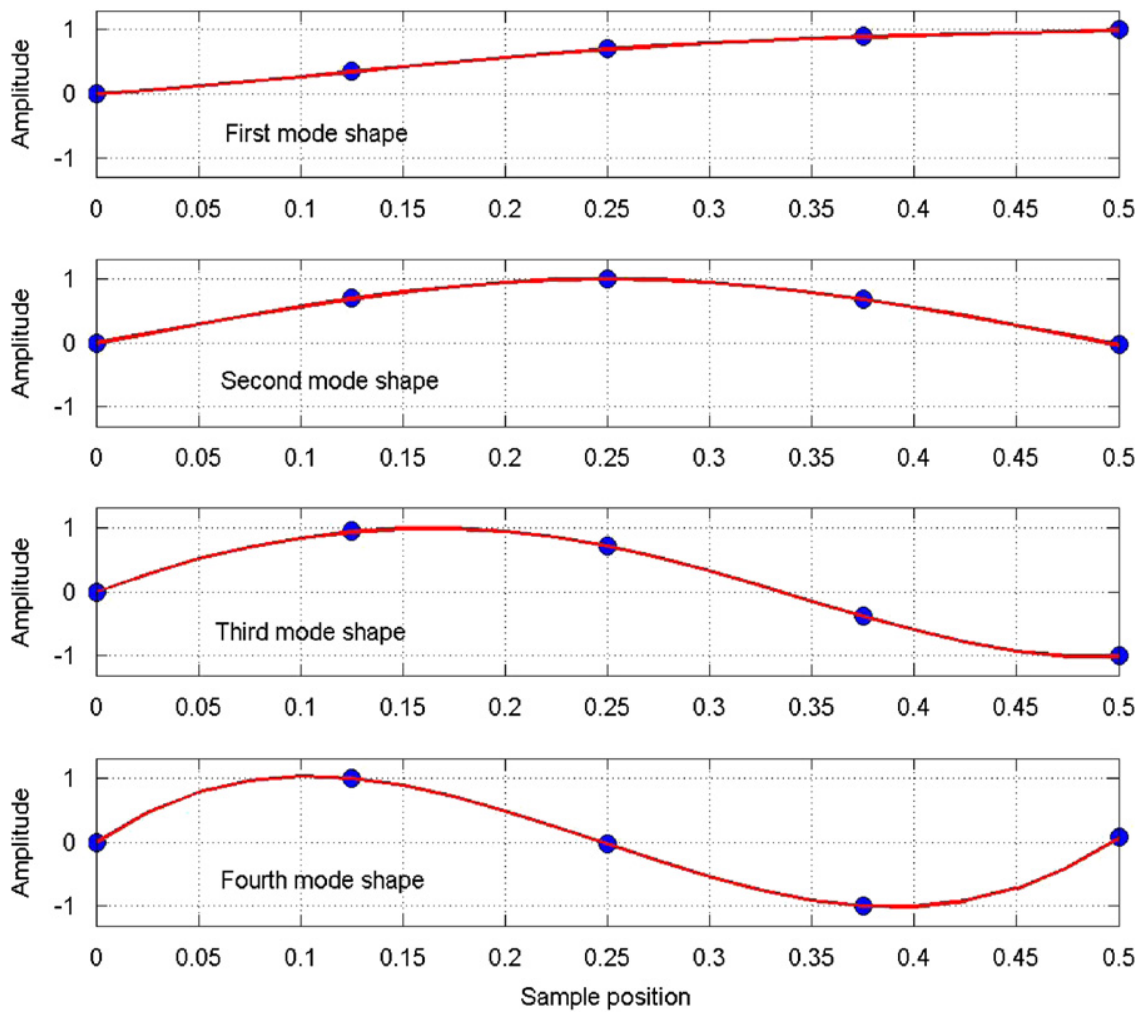

Fig. 4. Theoretical (solid line) and experimental (points) mode shapes for the line cable in laboratory. 
mode of vibration. The quantity

$$
\chi_{k i}(b)=\frac{\left(W_{\psi} x_{k}\right)\left(a_{i}, b\right)}{\left(W_{\psi} x_{r}\right)\left(a_{i}, b\right)}
$$

which is a function of time represents the $k$ th component of the $i$ th mode, normalized to the $r$ th component. By considering $m$ sensors and collecting the ratios evaluated by (55) a vector, which is a function of time, is obtained and is called instantaneous mode shape of the $i$ th mode of vibration

$$
\chi_{i}(b)=\left[\chi_{1 i}(b) \chi_{2 i}(b) \ldots \chi_{m i}(b)\right]
$$

The WT method requires a previous choice of the value of the dilatation parameter $a_{i}$ corresponding to the analyzed mode and the resolution of the wavelet transform depends on the value of this scale parameter, thus the choice of the analyzing wavelet is important.

\section{Applications}

\subsection{Modal parameter identification of a line cable in laboratory}

To prove the effectiveness of the identification procedure based on the subspace analysis and on the wavelet transform, we analyze the dynamic behaviour of transmission lines through the comparison of theoretical and experimental results.

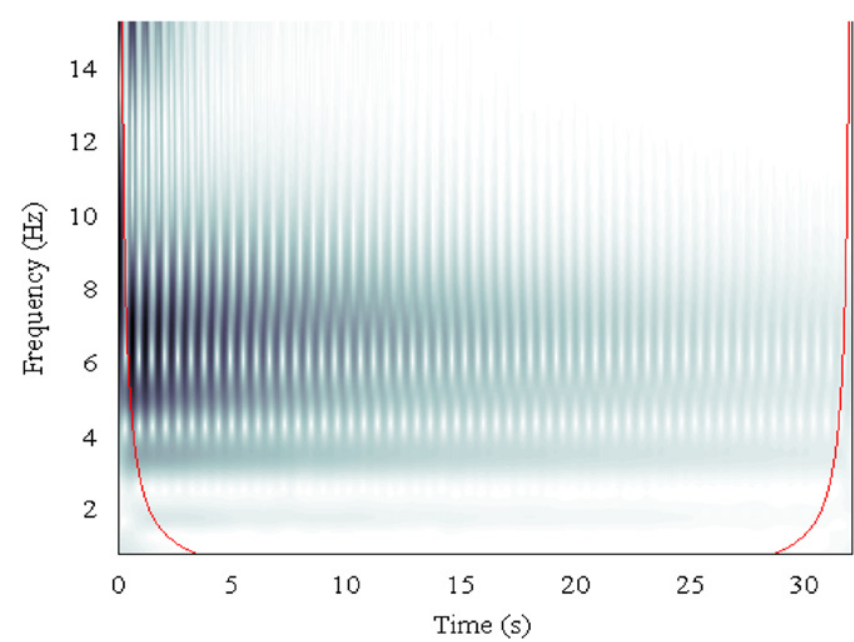

Fig. 5. Wavelet transform amplitude for the experimental line cable in laboratory with $N=2$.

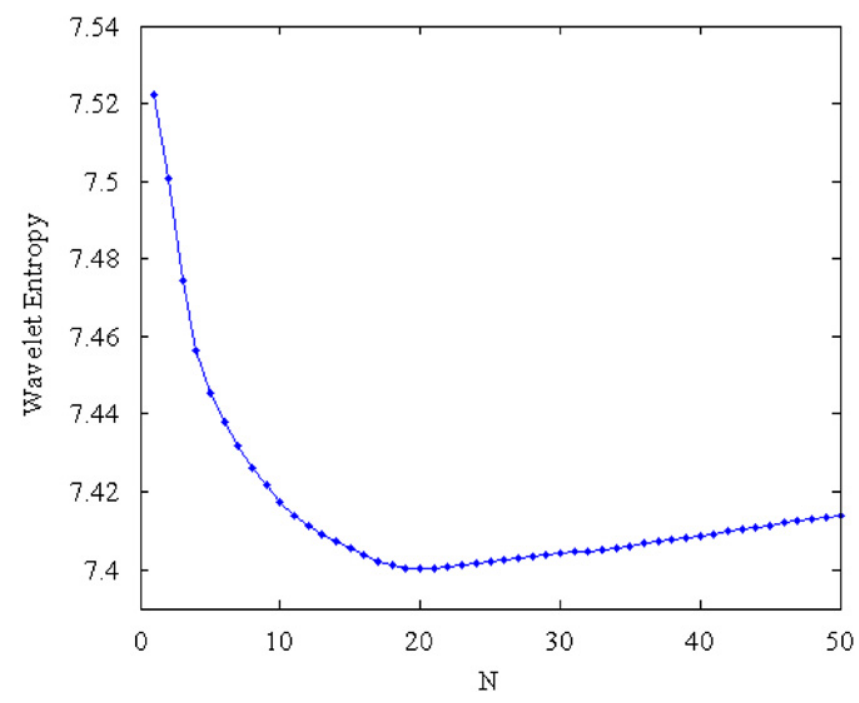

Fig. 6. Variations of the wavelet entropy for the line cable in laboratory. 
The schematic view of the testing system is shown in Fig. 1 and obtained from Barbieri et al. [16,17]. The parameters of the cable are: specific mass $0.8127 \mathrm{~kg} / \mathrm{m}$ and rigidity flexural $(E I) 11.07 \mathrm{Nm}^{2}$. For the modal identification four accelerometers are placed in the cable in the position $L / 2,3 L / 8, L / 4$ and $L / 8$. The length of the cable is $L=32.3 \mathrm{~m}$ and the mechanical load is $10,700 \mathrm{~N}$. The excitation of the cable is obtained through an impact hammer at $5.38 \mathrm{~m}$ of the extremity of the cable and Fig. 2 shows the time responses of different accelerometers. The recording time is $32 \mathrm{~s}$ and the sampling frequency is $64 \mathrm{~Hz}$. We have then 2048 points to form auto-covariance matrices. Before identification the data are filtered through a digital low-pass filter with a cut-off frequency of $30 \mathrm{~Hz}$. When performing modal analysis, one of the key decisions is to decide how many modes are there in the frequency range of interest. All the time domain modal identification algorithms have a serious problem on model order determination and when extracting physical or structural modes the subspace algorithm always generates spurious or computational modes to account for unwanted effects such as noise, leakage, residuals, nonlinearities... Practical experience in subspace modal analysis shows that it is better to overspecify the model order and to eliminate spurious poles afterwards. This can be done by constructing the stabilization diagram where models of increasing order are determined. Each model yields a set of modal parameters which are plotted in a stabilization diagram. In [1] the poles corresponding to a certain model order are compared to the poles of a one order lower model. If the eigenfrequency, the damping ratio and the related mode shape differences are within certain preset limits, the pole is labeled as a stable pole. In [1] the limits to obtain stable poles are $1 \%$ for eigenfrequencies, $5 \%$ for damping ratios and $1 \%$ for mode shape vectors. In this work, to eliminate spurious poles we have chosen a modal coherence indicator $\gamma_{k}$ superior to
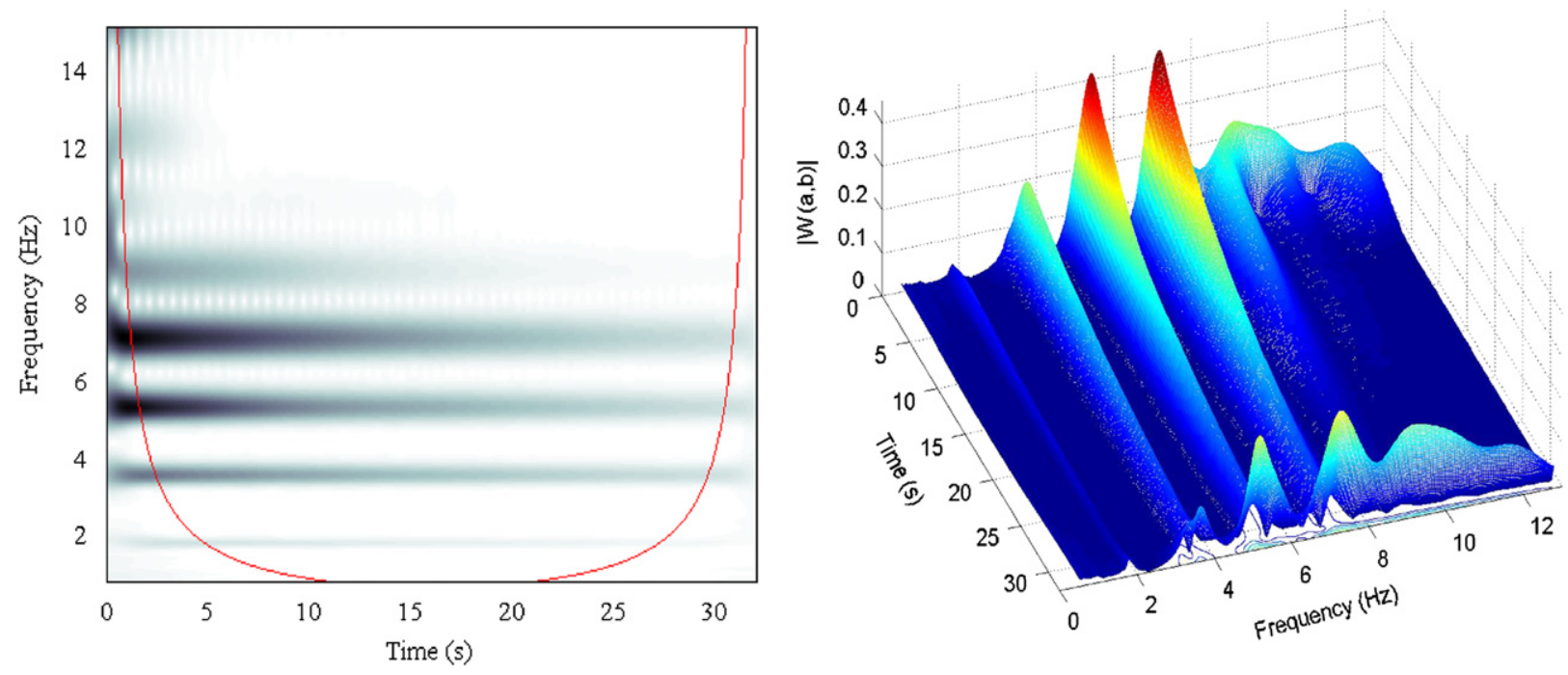

Fig. 7. Wavelet transform amplitude for the experimental line cable in laboratory with $N=20$.

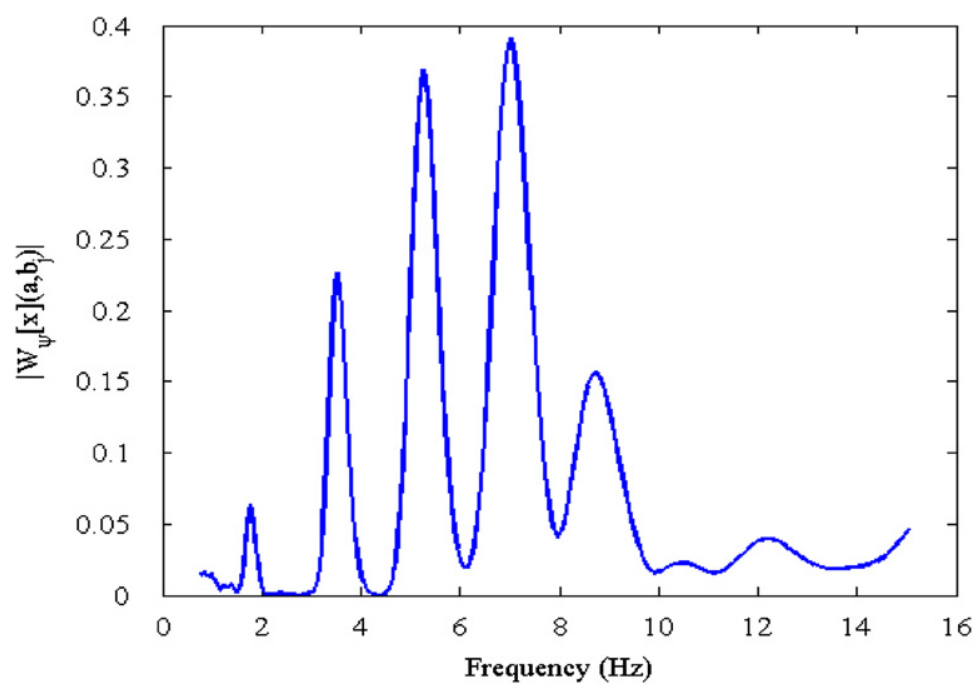

Fig. 8. Instantaneous wavelet spectra for the experimental line cable in laboratory. 
99\%. Fig. 3 shows the stabilization diagram on eigenfrequencies using the subspace method and the modal coherence indicator. A definition of the stabilization diagram is presented. The true system order is unknown and a common practice in operational modal analysis is to calculate the modal parameters for increasing model orders. The stability diagram is then plotted where the estimates of eigenfrequencies are given as a function of model order. In other words, the stability diagram involves tracking the estimates of eigenfrequencies as a function of model order. For modes that are very active in the measured data, the modal parameters stabilize at a very low model order and for modes that are poorly excited in the measured data, the modal parameters may not stabilize until a very high model order is reached. Nevertheless, the non-physical or computational modes do not stabilize at all during this process and can be sorted out of the modal parameters data set using the modal coherence indicator. The detection of eigenfrequencies is performed visually in a socalled stabilization diagram. The stabilization diagram shows very stable eigenfrequencies and from this plot (Fig. 3 ) we obtain the experimental natural frequencies of the cable. Table 1 shows the theoretical and experimental natural frequencies and damping ratios of the cable using different configurations of sensors. The theoretical natural frequencies are obtained from (2) and the experimental modal parameters are obtained using (12) and (13). Fig. 4 shows the
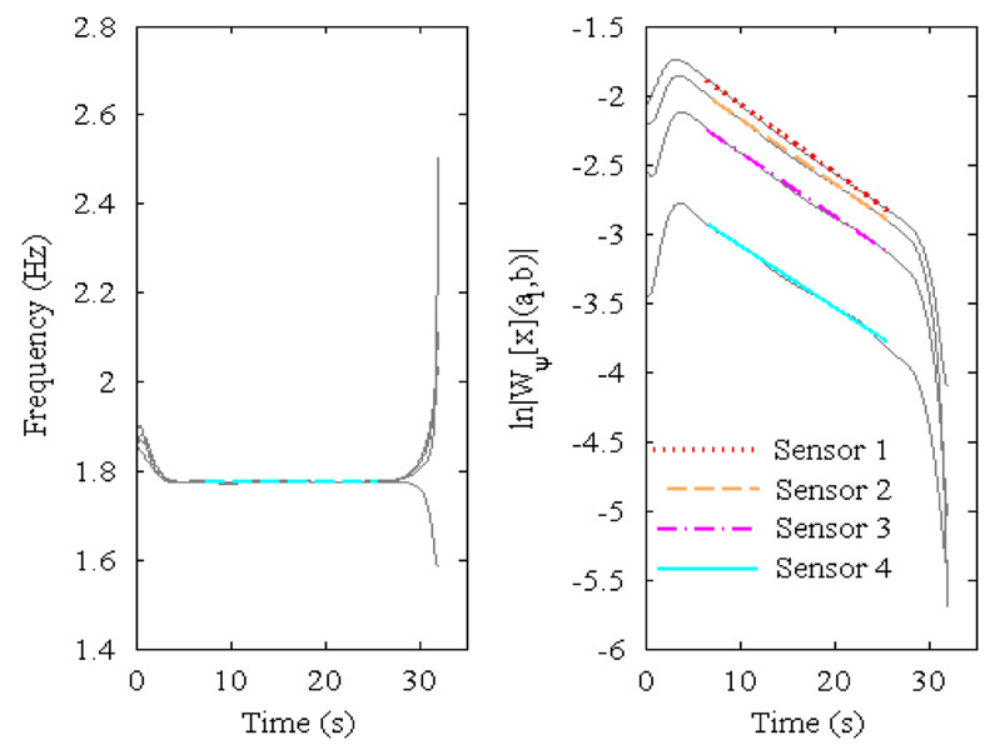

Fig. 9. Instantaneous frequency and envelope of the wavelet transform for the first mode of the experimental line cable.
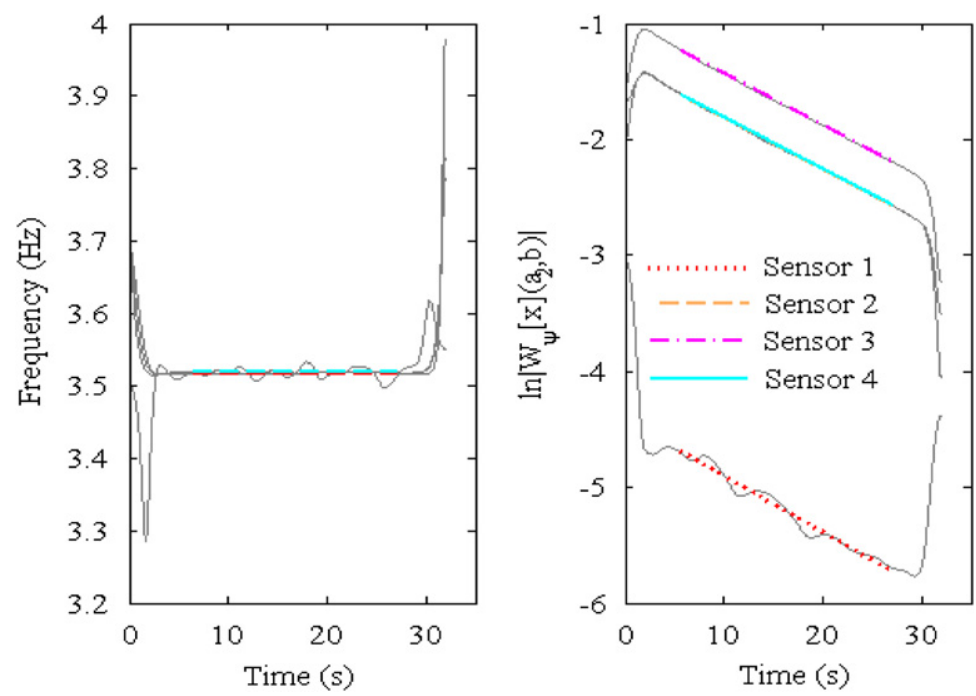

Fig. 10. Instantaneous frequency and envelope of the wavelet transform for the second mode of the experimental line cable in laboratory. 
theoretical (solid line) and experimental (points) mode shapes of the vibrating cable. From all these results, it can be seen that good estimates of modal parameters are obtained from the subspace algorithm, using output-only measurements. The modal parameters of the cable are also obtained by the WT method. However, if we use the traditionally Morlet wavelet with $N=2$ we obtain inexploitable results presented in Fig. 5; we cannot extract rigorously the eigenfrequencies and present modes are impossible to observe. The resolution of the wavelet transform is not sharp enough to separate different modes of the system. The modified Morlet wavelet is then used and the optimal value of the parameter $N$ is obtained by minimisation of the wavelet entropy. It shown in Fig. 6 that $N_{\text {optimal }}=20$. Fig. 7 shows the WT amplitude in 2D and 3D representations. The resolution of the wavelet transform is now accurate enough and present modes can be easily observed. The first five modes are visible from these plots, and the eigenfrequencies can be obtained directly from Fig. 8 representing the instantaneous wavelet spectra for the experimental line cable in laboratory. The modal parameters
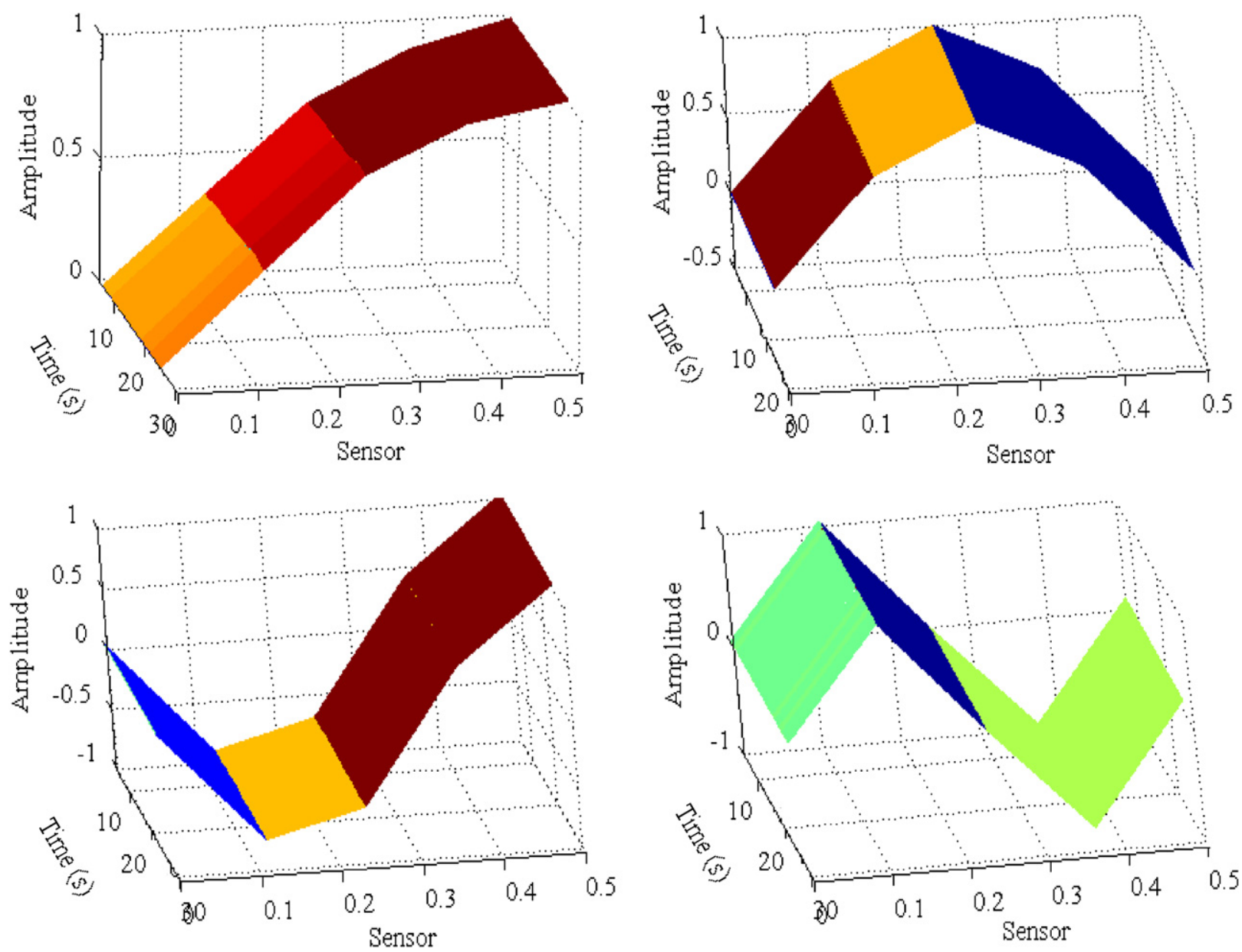

Fig. 11. The first four instantaneous mode shapes for the experimental line cable in laboratory.

Table 2

Natural frequencies and damping ratios of the experimental cable using the wavelet transform method.

\begin{tabular}{|c|c|c|c|c|c|c|c|c|}
\hline \multirow[t]{2}{*}{ Modes } & \multicolumn{2}{|c|}{ Sensor 1} & \multicolumn{2}{|c|}{ Sensor 2} & \multicolumn{2}{|c|}{ Sensor 3} & \multicolumn{2}{|c|}{ Sensor 4} \\
\hline & $f(\mathrm{~Hz})$ & $\zeta(\%)$ & $f(\mathrm{~Hz})$ & $\zeta(\%)$ & $f(\mathrm{~Hz})$ & $\zeta(\%)$ & $f(\mathrm{~Hz})$ & $\zeta(\%)$ \\
\hline 1 & 1.776 & 0.465 & 1.775 & 0.455 & 1.776 & 0.452 & 1.776 & 0.432 \\
\hline 2 & 3.517 & 0.234 & 3.518 & 0.210 & 3.518 & 0.208 & 3.518 & 0.212 \\
\hline 3 & 5.255 & 0.244 & 5.255 & 0.242 & 5.255 & 0.258 & 5.256 & 0.251 \\
\hline 4 & 7.018 & 0.145 & 7.018 & 0.143 & 7.018 & 0.117 & 7.018 & 0.146 \\
\hline 5 & 8.734 & 0.189 & 8.734 & 0.192 & 8.734 & 0.190 & 8.734 & 0.187 \\
\hline
\end{tabular}


(eigenfrequencies and damping ratios) are obtained using (50) and (51) and from the plots shown in Figs. 9 and 10 where only the two first modes are represented. Finally, the first instantaneous mode shapes of the cable obtained from (55) and (56) are represented in Fig. 11. Table 2 presents the modal parameters obtained by the WT. A very good agreement can be found between the theoretical, the subspace and the WT methods in the natural frequencies estimation. The identification of the damping ratios, as usual, is most crucial. The differences between the results obtained by the subspace method and the WT method are due to the presence of some spurious modes in the stabilization diagram, which could lead to erroneous results, the edge effects in the WT, the nature of the input forces exciting the cable: all modes are not excited in the similar way and finally the presence of nonlinearities.

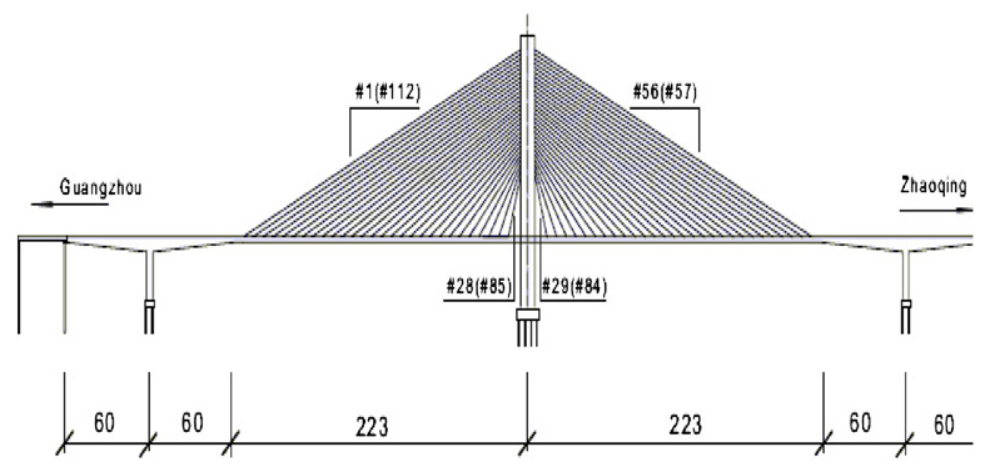

(m)

Fig. 12. Schematic view of the Jinma bridge with stay cables.

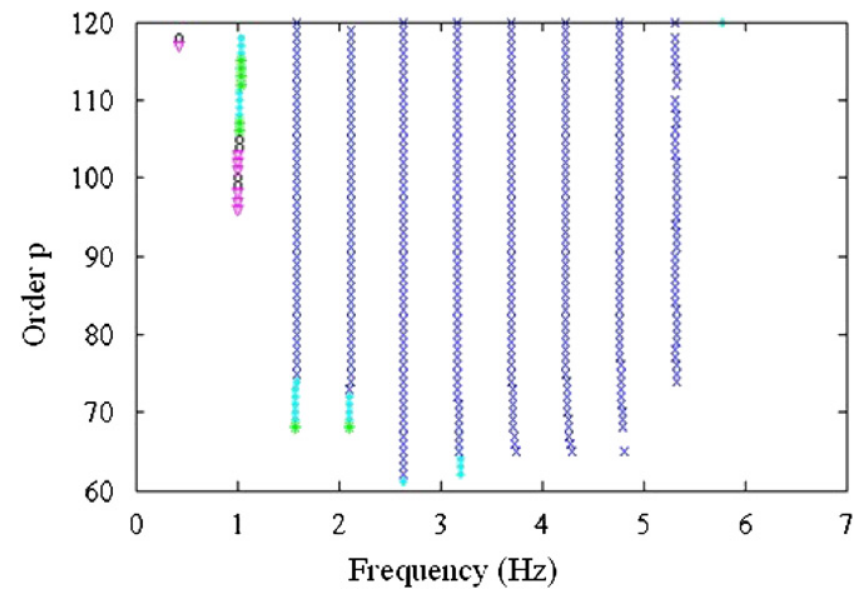

Fig. 13. Stabilization diagram on eigenfrequencies for the first stay cable of the bridge (Cable 1).

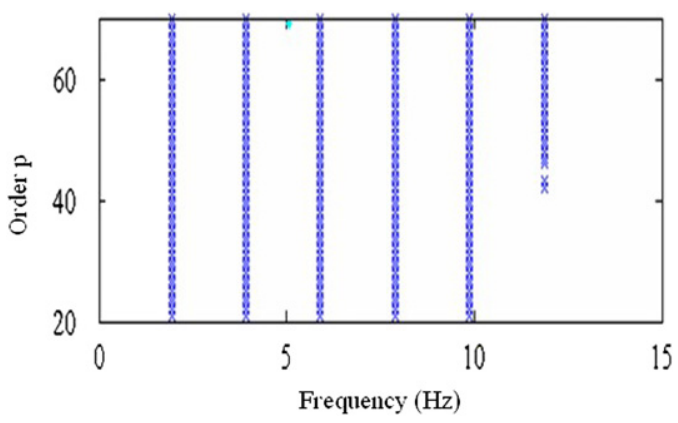

Fig. 14. Stabilization diagram on eigenfrequencies for the cable 25 of the bridge. 


\subsection{Modal parameter identification of the Jinma cable-stayed bridge}

The subspace method and the wavelet transform method are applied to the analysis of stay cables of the Jinma cablestayed bridge (Fig. 12), that connects Guangzhou and Zhaoqing in Guangdong Province, China. It is a single tower, double row cable-stayed bridge, supported by $28 \times 4=112$ stay cables. Before the official opening of the bridge, ambient vibration tests on each stay cable were carried out for the purpose of cable tension evaluation. Inputs could evidently not be measured, so only acceleration data are available. For the ambient vibration measurement of each stay cable an accelerometer was mounted securely to the cables, the sampling frequency is $40 \mathrm{~Hz}$ and the recording time is $140.8 \mathrm{~s}$,

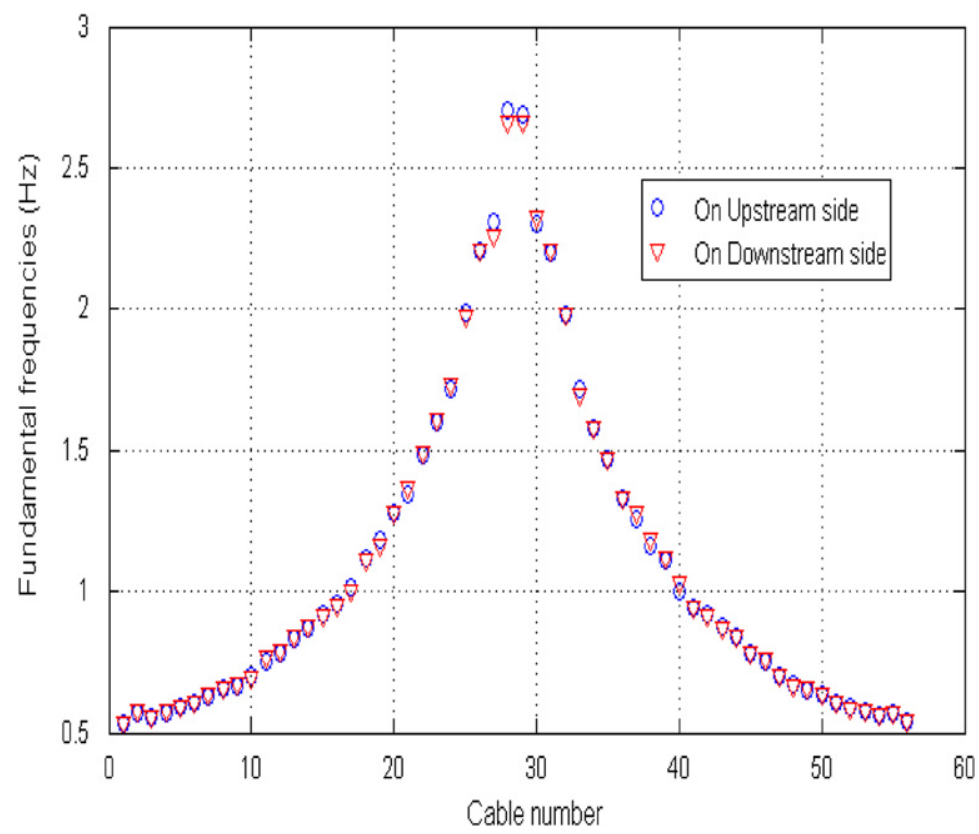

Fig. 15. Fundamental frequency of each cable on upstream side and on downstream side.

a

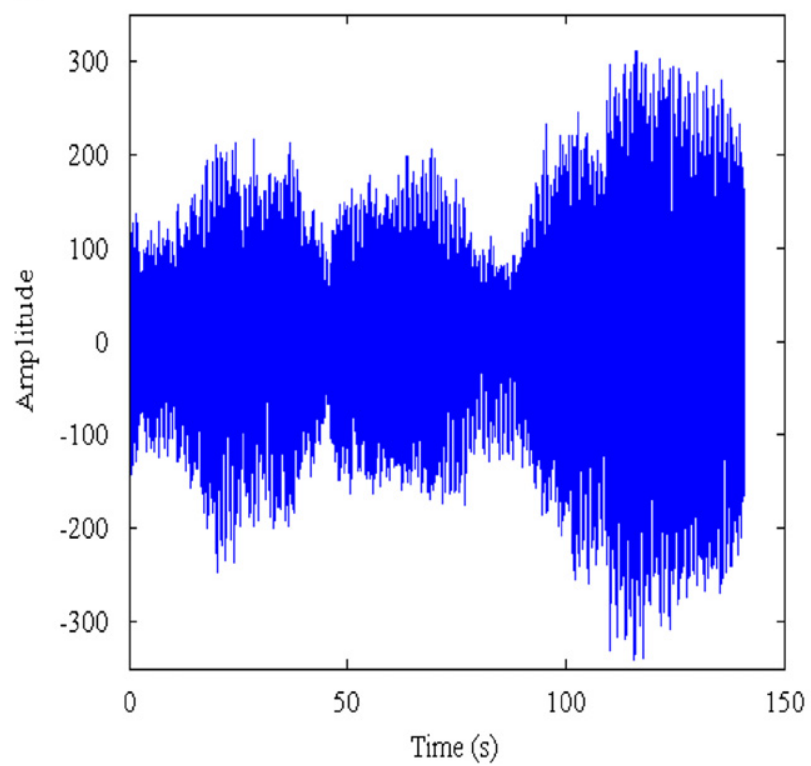

b

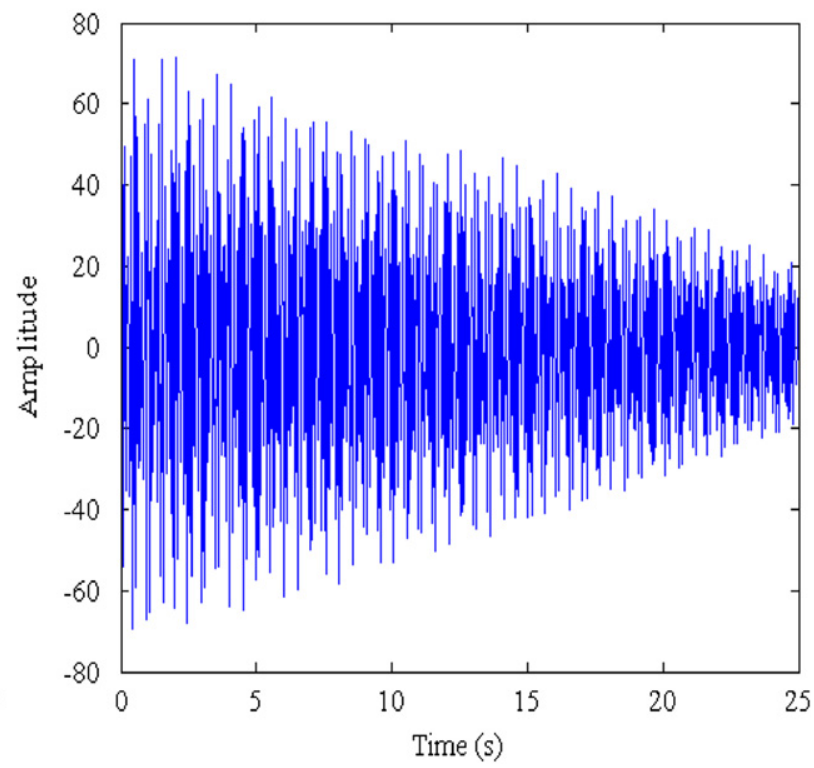

Fig. 16. (a) Ambient response of cable 25: amplitude $\left(\mathrm{mm} \mathrm{s}^{-2}\right.$ ) versus time (s) and (b) random decrement signature of cable 25 : amplitude (mm $\mathrm{s}^{-2}$ ) versus time $(\mathrm{s})$. 
Table 3

Natural frequencies, damping ratios and Scruton number of cable 25 using the subspace method.

\begin{tabular}{lllrrr}
\hline Modes & 1 & 2 & 3 & 4 & 5 \\
\hline$f(\mathrm{~Hz})$ & 1.965 & 3.932 & 5.905 & 7.886 & 9.876 \\
$\zeta(\%)$ & 0.269 & 0.035 & 0.160 & 0.066 & 0.070 \\
$S_{c}$ & 3.641 & 0.473 & 2.165 & 0.893 & 0.947 \\
\hline
\end{tabular}

Table 4

Natural frequencies, damping ratios and Scruton number of cable 25 using the wavelet transform method.

\begin{tabular}{|c|c|c|c|c|c|c|}
\hline Modes & 1 & 2 & 3 & 4 & 5 & 6 \\
\hline$f(\mathrm{~Hz})$ & 1.960 & 3.938 & 5.915 & 7.893 & 9.856 & 11.884 \\
\hline$\zeta(\%)$ & 0.314 & 0.046 & 0.178 & 0.078 & 0.081 & 0.094 \\
\hline$S_{c}$ & 4.250 & 0.622 & 2.410 & 1.056 & 1.096 & 1.271 \\
\hline
\end{tabular}

which results in total 5632 data points. Cables 1, 56, 57 and 112 are the longest and cables 28, 29, 84 and 85 are the shortest. A full description of the test can be found in [18]. Figs. 13 and 14 show the stabilization diagrams on eigenfrequencies of cables 1 and 25 using the subspace method and the modal coherence indicator. These diagrams show remarkable stable eigenfrequencies and from these plots we determine the eigenfrequencies of the two stay cables. In fact, we apply the subspace method to each of 120 stay cables to obtain the fundamental frequency $f_{0}$ of each cable. The fundamental frequencies of all 112 stay cables determined via subspace analysis of ambient vibration responses for upstream stay cables and downstream stay cables are presented in Fig. 15. It can be seen that the fundamental frequencies of both bridge sides are almost identical and the fundamental frequency distribution is symmetric with respect to the single tower. The fundamental frequencies vary between $0.533 \mathrm{~Hz}$ for the longest cable and $2.703 \mathrm{~Hz}$ for the shortest cable. The cable tension can be estimated by the approximated expression $T=4 \rho L^{2} f_{0}^{2}$, where $\rho$ is the linear density of the cable $(\rho=66.94 \mathrm{~kg} / \mathrm{m}), L$ the length of the cable and $f_{0}$ the fundamental frequency. The maximum and minimum cable forces for the Jinma bridge are then: $T_{\max }=5052.073 \mathrm{kN}$ (cable no. 57), $T_{\min }=2490.653 \mathrm{kN}$ (cable no. 84). These cable forces can be considered as reference tensions and used as indicators in the field of health monitoring.

The identification procedure of modal parameters using the WT is carried out as previously. At first we apply the random decrement technique to the cable no. 25 to obtain its free response (see Fig. 16). We apply then the WT method to this free response to obtain the modal parameters of this stay cable. Tables 3 and 4 show the values of modal parameters of cable 25 using the subspace method and the WT method. A very good agreement can be found between the two methods in the natural frequency estimation. The identification of the damping ratios differs slightly. The differences are due to the presence of some spurious modes in the stabilization diagram; the use of the random decrement method to obtain the free response of the cable, the edge effects in the WT, the nature of the input forces exciting the cable and the presence of nonlinearities.

The instability of a cable is assessed by the Scruton number [21], which is defined for each mode of vibration by $S_{c, i}=\frac{\zeta_{i} \rho}{\rho_{a} D^{2}}$, where $\zeta_{i}$ is the damping ratio for each mode, $\rho$ the mass of the cable per meter, $\rho_{a}$ the density of air and $D$ the cable diameter. High values of the Scruton number tend to suppress the oscillation and bring up the start of instability at high wind speeds. Considering $\rho=66.94 \mathrm{~kg} / \mathrm{m}, \rho_{a}=1.2 \mathrm{~kg} / \mathrm{m}^{3}$ and $D=0.203 \mathrm{~m}$, the Scruton number for each mode is presented in Tables 3 and 4. In the Scruton number damping is a main factor and most vibration problems in stay cables are subject to low damping values. The situation can be resolved by the improvement of damping through devices which are fixed to cables. A very economic and effective solution is the application of connecting wires between neighbouring cables in order to change the vibration system as well as increase damping considerably.

\section{Conclusion}

Modal parameter identification of stay cables in a cable-stayed bridge is a typical class of structural modal analysis and it is of practical significance to develop methods for identifying modal parameters with only measured response data. In this paper, it is shown that the subspace and the WT transform methods can be effectively employed in operational modal analysis. The output-only measurement techniques have already demonstrated their robustness and reliability when applied to ambient vibrations especially in eigenfrequencies identification of stay cables. The subspace method is improved by the introduction of a modal coherence indicator which eliminates spurious modes. The WT method uses a modified Morlet wavelet where a coefficient is obtained by entropy minimisation. Once the eigenfrequencies and the damping coefficients have been identified, the cable forces and the Scruton number are derived. In a continuous monitoring and modal analysis process, the tension forces and Scruton numbers could be used to assess the health of stay cables in cablestayed bridges. Operational modal analysis applied to the dynamic data of stay cables provides useful information to determine the current condition of stay cables accurately. 
Large structures tend to present large motions, and therefore nonlinearities. The use of the wavelet transform for the identification of nonlinearities on damping and stiffness is under investigation. However, the concept established in the paper, where the hypothesis of linear vibrations is used, can be extended to nonlinear vibrations. Indeed, if we consider the response of a nonlinear oscillator and apply the wavelet transform, it is shown that the amplitude of the wavelet transform is related to nonlinear damping coefficients and the phase of the wavelet transform is related to nonlinear stiffness parameters. From the amplitude and the phase of the wavelet transform we can identify nonlinear damping and nonlinear stiffness in vibrating systems.

\section{Acknowledgments}

The authors would like to thank Dr. Barbieri at the Pontificia Universidade Catolica do Parana (Brazil) and Dr. Ren at the University of Fuzhou (People's Republic of China) for generously providing the data for this study.

\section{References}

[1] B. Peeters, G. De Roeck, Reference based stochastic subspace identification for output-only modal analysis, Mechanical Systems and Signal Processing 13 (1999) 855-878.

[2] R. Brincker, L. Zhang, P. Andersen, Modal identification of output-only systems using frequency domain decomposition, Smart Materials and Structures 10 (2001) 441-445.

[3] J. Lardies, A stochastic realization algorithm with application to modal parameter estimation, Mechanical Systems and Signal Processing 15 (2001) 275-285.

[4] K.A. Petsounis, S.D. Fassois, Parametric time-domain methods for the identification of vibrating structures-a critical comparison and assessment, Mechanical Systems and Signal Processing 15 (2001) 1031-1060.

[5] J.B. Bodeux, J.C. Golinval, Modal identification and damage detection using the data-driven stochastic subspace and ARMAV methods, Mechanical Systems and Signal Processing 17 (2003) 83-89.

[6] Y. Zhang, Z. Zhang, X. Xu, H. Hua, Modal parameter identification using response data only, Journal of Sound and Vibration 282 (2005) 367-380.

[7] J.N. Juang, in: Applied System Identification, Prentice Hall, New-Jersey, USA, 1994.

[8] L. Zhang, Modal indicators for operational modal identification, in: Proceedings of the 19th IMAC, Orlando, Florida, 2001.

[9] C. Gontier, Energetic classifying of vibration modes in subspace stochastic modal analysis, Mechanical Systems and Signal Processing 19 (2005) 1-19.

[10] C. CHUI, in: An Introduction to Wavelets, Academic Press, New York, 1995.

[11] W.J. Staszewski, Identification of damping in MDOF systems using time-scale decomposition, Journal of Sound and Vibration 203 (1997) 283-305.

[12] M. Ruzzene, A. Fasana, L. Garibaldi, B. Piombo, Natural frequencies and dampings identification using the wavelet transform: application to real data Mechanical Systems and Signal Processing 11 (1997) 207-218.

[13] T.-P. Le, P. Argoul, Continuous wavelet transform for modal identification using free decay response, Journal of Sound and Vibration 277 (2004) $73-100$.

[14] J. Lardies, M.N. TA, M. Berthillier, Modal parameter estimation based on the wavelet transform of output data, Archives of Applied Mechanics 73 (2004) 718-733.

[15] S.R. Ibrahim, Random decrement technique for modal identification structures, Journal of Spacecraft and Rockets 14 (1977) 696-700.

[16] N. Barbieri, O. Honorato De Souza Junior, R. Barbieri, Dynamical analysis of transmission line cables. Part $1-$ linear theory, Mechanical Systems and Signal Processing 18 (2004) 659-669.

[17] N. Barbieri, O. Honorato De Souza Junior, R. Barbieri, Dynamical analysis of transmission line cables. Part 2-damping estimation, Mechanical Systems and Signal Processing 18 (2004) 671-681.

[18] W. X. Ren, G. Chen, Experimental modal analysis of stay cables in cable-stayed bridges, in: Proceedings of the 21st IMAC, Kissimmee, Florida, 2003.

[19] S.S Rao, in: Mechanical Vibrations, Addison Wesley Publishing Company, 1995.

[20] A. SPATARU, in: Fondements de la théorie de la transmission et de l'information, Presses Polytechniques Romandes, Lausanne, 1987.

[21] R. Geier, R. Flesh, Cable-stayed bridges and their dynamic response, in: Proceedings of the 22nd IMAC, Detroit, Michigan, 2004. 